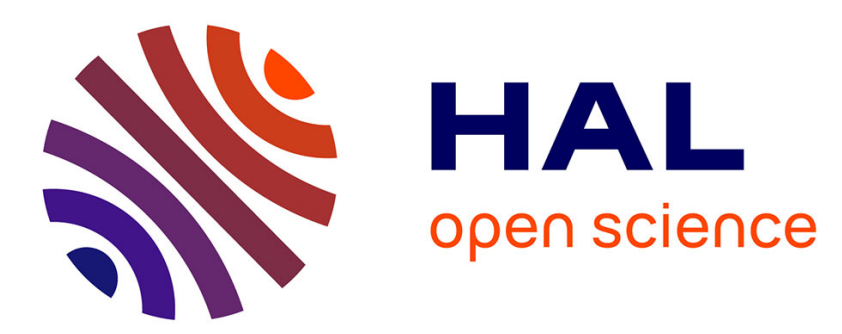

\title{
Compound channel flow with a longitudinal transition in hydraulic roughness over the floodplains
}

\author{
V. Dupuis, Sébastien Proust, Céline Berni, André Paquier
}

\section{To cite this version:}

V. Dupuis, Sébastien Proust, Céline Berni, André Paquier. Compound channel flow with a longitudinal transition in hydraulic roughness over the floodplains. Environmental Fluid Mechanics, 2017, 17 (5), pp.903-928. 10.1007/s10652-017-9525-0 . hal-01807794

\section{HAL Id: hal-01807794 \\ https://hal.science/hal-01807794}

Submitted on 5 Jun 2018

HAL is a multi-disciplinary open access archive for the deposit and dissemination of scientific research documents, whether they are published or not. The documents may come from teaching and research institutions in France or abroad, or from public or private research centers.
L'archive ouverte pluridisciplinaire HAL, est destinée au dépôt et à la diffusion de documents scientifiques de niveau recherche, publiés ou non, émanant des établissements d'enseignement et de recherche français ou étrangers, des laboratoires publics ou privés. 


\title{
Compound channel flow with a longitudinal transition in hydraulic roughness over the floodplains
}

\author{
Victor Dupuis · Sébastien Proust · Céline \\ Berni · André Paquier
}

Received: date / Accepted: date

\begin{abstract}
Flows in a compound open-channel (two-stage geometry with a main channel and adjacent floodplains) with a longitudinal transition in roughness over the floodplains are experimentally investigated in an $18 \mathrm{~m}$ long and $3 \mathrm{~m}$ wide flume. Transitions from submerged dense vegetation (meadow) to emergent rigid vegetation (wood) and vice versa are modelled using plastic grass and vertical wooden cylinders. For a given roughness transition, the upstream discharge distribution between main channel and floodplain (called subsections) is also varied, keeping the total flow rate constant. The flows with a roughness transition are compared to flows with a uniformly distributed roughness over the whole length of the flume. Besides the influence of the downstream boundary condition, the longitudinal profiles of water depth are controlled by the upstream discharge distribution. The latter also strongly influences the magnitude of the lateral net mass exchanges between subsections, especially upstream from the roughness transition. Irrespective of flow conditions, the inflection point in the mean velocity profile across the mixing layer is always observed at the interface between subsections. The longitudinal velocity at the main channel/floodplain interface, denoted $U_{i n t}$, appeared to be a key parameter for characterising the flows. First, the mean velocity profiles across the mixing layer, normalised using $U_{\text {int }}$, are superimposed irrespective of downstream position, flow depth, floodplain roughness type and lateral mass transfers. However, the profiles of turbulence quantities do not coincide, indicating that the flows are not fully self-similar and that the eddy viscosity assumption is not valid in this case. Second, the depth-averaged turbulent intensities and Reynolds stresses, when scaled by the depth-averaged velocity $U_{d, \text { int }}$ exhibit two plateau values, each related to a roughness type, meadow or wood. Lastly, the same results hold when scaling by $U_{d, \text { int }}$ the depth-averaged lateral flux of momentum due to secondary currents. Turbulence
\end{abstract}

V. Dupuis · S. Proust · C. Berni · A. Paquier

Irstea, UR HHLY Hydrologie-Hydraulique, Centre de Lyon-Villeurbanne

5 rue de la Doua BP32108

F-69616 Villeurbanne cedex, France

Tel.: +33-47-2208787

Fax: $+33-47-8477875$

E-mail: victor.dupuis@centraliens-lille.org·sebastien.proust@irstea.fr·celine.berni@irstea.fr · andre.paquier@irstea.fr 
production and magnitude of secondary currents are increased by the presence of emergent rigid elements over the floodplains. The autocorrelation functions show that the length of the coherent structures scales with the mixing layer width for all flow cases. It is suggested that coherent structures tend to a state where the magnitude of velocity fluctuations (of both horizontal vortices and secondary currents) and the spatial extension of the structures are in equilibrium.

Keywords Laboratory study · Non-uniform flow • Turbulent mixing layer . Coherent structures $\cdot$ Cylinder array $\cdot$ Rigid vegetation

\section{Introduction}

During high flood events, the flow in the main channel of the river overflows the adjacent floodplains, and the resulting flow takes place in a compound channel, a two-stage geometry. Compound open channel flows have been widely investigated under uniform flow conditions (e.g. Knight and Demetriou, 1983; Tominaga and Nezu, 1991). However, these flows are often subjected to different sources of flow nonuniformity in the field. A first source is the longitudinal variation in cross-sectional shape, which was studied for example in the case of skewed floodplains (Elliott and Sellin, 1990), of narrowing or enlarging floodplains (Bousmar et al, 2004; Proust et al, 2010), of local obstacles like groynes set on the floodplains (Peltier et al, 2013). These studies showed that the lateral momentum exchange between the main channel flow and the floodplain flow is driven by both the turbulent diffusion related to the mixing layer that forms at the main channel/floodplain interface and by the net lateral mass transfers between main channel and floodplain. Proust et al (2013) investigated the relaxation of flows in a straight smooth compound channel in which the upstream discharge distribution between main channel and floodplain was destabilized with regards to the uniform discharge distribution. It appears that the relaxation to uniformity is a relatively slow process: for an excess or a deficit in floodplain discharge of $\pm 19 \%$ at the flume inlet, the uniform discharge distribution is not reached $10 \mathrm{~m}$ downstream. Bousmar et al (2005) pointed out that the longitudinal development of a compound channel mixing layer is slower than the development of a vertical boundary layer.

Longitudinal changes in hydraulic roughness over the floodplain are also an important source of flow non-uniformity. Indeed, changes in land occupation are common in the field, such as transitions from meadows or cultivated lands to woodland or urbanized areas. In these cases, a transition from a bed roughness to emergent roughness elements can be observed. The transition zones between two reaches with such different roughness types, where water depth and/or cross-sectional discharge distribution vary, can be expected to be characterised by a complex flow. The objective of the present paper is to gain insight into the flow processes in such regions.

Compound channel flows with a longitudinal transition in roughness were scarcely investigated so far. Jahra et al (2011) investigated compound channel flows through short patches of emergent rigid vegetation on the floodplain and successfully reproduced their experimental results with a $3 \mathrm{D}$ numerical simulation. Compound channel flows with a longitudinal change in roughness can be compared to flows in a channel with a rectangular cross-section (called herein single channel) where a longitudinal and a lateral transition in roughness are combined. In this case, the lateral change 
in roughness also induces a mixing layer. Such flows were investigated by Vermaas et al (2011) in the case of a rough bed parallel to a smooth bed, by Rominger and Nepf (2011) in the case of a patch of emergent cylinders of finite width in the middle of a flat channel, and by Zong and Nepf (2010) in the case of an emergent cylinder array occupying the right third of the channel width. Vermaas et al (2011) showed that the contribution of secondary currents to the lateral exchange of momentum between the two beds can be of the same order of magnitude than the contribution of net mass transfers and of turbulent diffusion. Rominger and Nepf (2011) showed that the patch influences the flow over a length of $L_{u p} \approx 4 b$ upstream of the patch, where $b$ is the patch half-width. Downstream of the leading edge, they defined an interior adjustment region, associated with net lateral mass transfers, followed by a region of vortex growth, before the flow gets fully developed with a constant vortex size.

The present study investigates compound channel flows subjected to a longitudinal transition in hydraulic roughness over the floodplains. The transition occurs between a rough bed, representing a submerged dense meadow, and an array of emergent cylinders, representing a woodland with emergent trees. As pointed out in Dupuis et al (2017), compound channel flows with either a bed roughness or a cylinder array on the floodplain feature, for the same total discharge, different cross-sectional distributions of longitudinal momentum, of secondary currents and of turbulent quantities. In addition, if the mixing layer develops self-similarly all along the flume for both roughness types, its growth rate significantly differs from one type to another. Lastly, it was shown that flow uniformity was not reached at the channel end even with a homogeneous roughness on the entire floodplain. As the longitudinal flow development occurs over a large distance in the case of uniform floodplain roughness, the following questions arise: what happens in the case of a spatial transition between two different types of roughness? How behaves the mixing layer at the transition?

The main objective is here to study the compound channel mixing layer properties under non-uniform flow conditions, i.e. in the presence of longitudinal water depth gradients and of lateral net mass exchanges between main channel and floodplain. In a previous study, we investigated the same roughness transitions using the same experimental facility in a single channel configuration, to model a floodplain isolated from the main channel (Dupuis et al, 2016). It was shown that, upstream of the transition, the water depth was varying and the vertical profiles of velocity and turbulence quantities were self-similar; by contrast, downstream of the transition, the water depth was constant and velocity and turbulence quantities were no more self-similar.

The experimental setup and flow configurations are exposed in Section 2. The investigated flows are first described from a macroscopic point of view: the longitudinal variation in water depth is presented in Section 3 and the discharge distribution between main channel and floodplain in Section 4 . The mixing layer dynamics is then studied in Section 5, the coherent structures in Section 6, the turbulent exchange of momentum at the interface between main channel and floodplain in Section 7, and finally the secondary currents in the main channel in Section 8. 
(a)

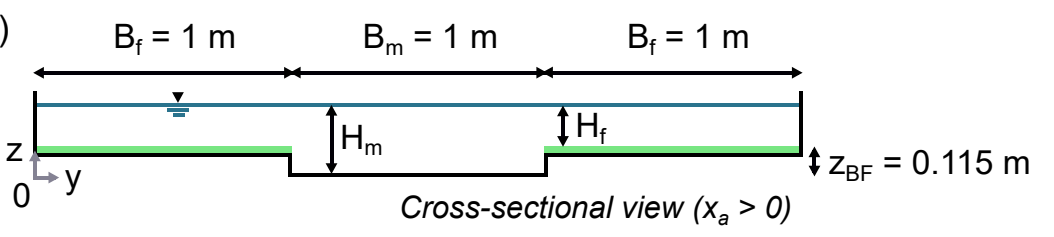

(b)

$\stackrel{0.75 \mathrm{~m}}{\longleftrightarrow} 17.25 \mathrm{~m}$

$\mathrm{CM}$

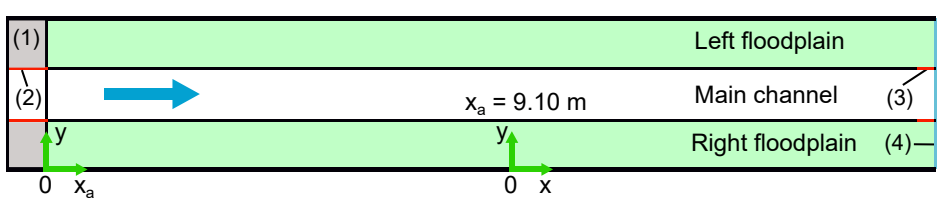

$\mathrm{CW}$
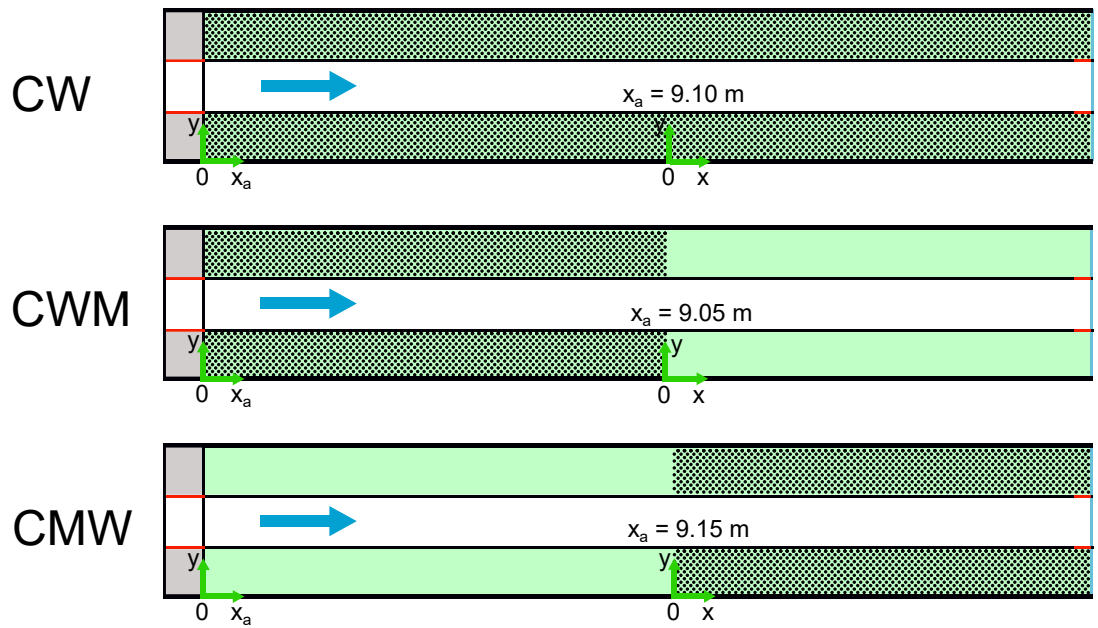

Fig. 1: (a) Cross-sectional view of the compound channel flume. (b) Plan view of the four flow configurations: (1) floodplain inlet ramp, (2) upstream splitter plate, (3) downstream splitter plate, (4) downstream weir.

\section{Experimental setup and methodology}

The experiments were performed in an $18 \mathrm{~m}$ long and $3 \mathrm{~m}$ wide glassed-wall flume, located in the Hydraulics and Hydromorphology Laboratory of Irstea, LyonVilleurbanne, France. The compound channel cross section was symmetrical and composed of a rectangular central main channel of width $B_{m}=1 \mathrm{~m}$ and of two adjacent floodplains of width $B_{f}=1 \mathrm{~m}$ (Fig. 1a). The longitudinal bottom slope was $S_{0}=1.05 \mathrm{~mm} . \mathrm{m}^{-1}$. Both right and left floodplains were covered with a dense plastic grass with $5 \mathrm{~mm}$-long rigid blades. The bankfull level of the main channel, measured from the bottom of the main channel to the crest of the grass blades was $z_{B F}=115 \mathrm{~mm}$.

After leaving the inlet tanks, the flows in the right and left floodplains were accelerated along a ramp (Fig. 1b). The streams in the three subsections were sepa- 
rated by vertical splitter plates until the ramp end. The inlet discharges in the three subsections (main channel, right and left floodplains) were independently regulated with control valves and monitored by electromagnetic discharge-meters. The standard deviation of the discharge time series was of the order of $1.5 \%$ of the mean discharge value. A $100 \mathrm{~mm}$-thick honeycomb was installed in the inlet tank of the main channel to vertically and laterally homogenize the flow ( $8 \mathrm{~mm}$ alveolus). At the flume outlet, the flow was controlled by three independent weirs (one per subsection) and splitter plates maintained the flows in the three subsections separated over a distance of $50 \mathrm{~cm}$ upstream of the weirs.

The floodplains were covered either by plastic grass alone (meadow) or by an array of emergent cylinders set on the plastic grass (wood). Four flow configurations were investigated (Fig. 1b), corresponding to different floodplain land occupations: (1) floodplains covered by meadow along the whole flume length (configuration denoted CM for Compound channel with Meadow), (2) floodplains covered by wood along the whole length (CW: Compound channel with Wood), (3) floodplains covered by wood in the upstream half of the flume and by meadow in the downstream half (CWM: Compound channel with a longitudinal transition from Wood to Meadow) and (4) floodplains covered by meadow in the upstream half and by wood in the downstream half (CMW: Compound channel with a longitudinal transition from Meadow to Wood). The first two flow configurations (CM and CW) were investigated in detail in Dupuis et al (2017). Figure 2a shows a picture of configuration CWM.

The longitudinal axis ( $x$-axis) is defined along the flume bottom, the vertical axis $(z$-axis) is normal to the bottom and the lateral axis ( $y$-axis) is oriented from the right bank to the left bank. In this coordinate system, the instantaneous velocities, time-averaged velocities and velocity fluctuations are denoted $(u, v, w),(U, V, W)$ and $\left(u^{\prime}, v^{\prime}, w^{\prime}\right)$, respectively. Overline denotes time-averaging (e.g. $\left.\overline{u^{\prime} v^{\prime}}\right)$. Two longitudinal coordinates $x$ and $x_{a}$ are defined (see Fig. 1b). The $x_{a}$-origin is defined at the trailing edge of the upstream splitter plates. The $x$-origin is located at the roughness transition for the flows with a roughness transition $\left(x_{a}=9.05 \mathrm{~m}\right.$ for configuration CWM and at $x_{a}=9.15 \mathrm{~m}$ for configuration CMW) and at $x_{a}=9.10 \mathrm{~m}$ for the two uniform flows. In the following, all results are presented using the relative coordinate $x$. The origin of the lateral coordinate $y$ is located at the side wall of the right floodplain. The origin of the vertical coordinate $z$ is defined at the bottom of the main channel. A relative vertical coordinate $z_{f}$ is additionally defined, whose origin is located at the floodplain bottom (main channel bankfull level), such that $z_{f}=z-z_{B F}$. Subscripts $m$ and $f$ refer to main channel and floodplain, respectively.

The flow conditions of the six flows investigated are reported in Table 1. The total discharge was the same for all flow configurations $\left(Q_{t o t}=162 \mathrm{~L} . \mathrm{s}^{-1}\right)$. The boundary conditions were set differently for the flows with uniform roughness over the floodplain and for the flows with a roughness transition. (a) For test cases CM and CW, the upstream discharge distribution and the downstream weir levels were adjusted iteratively (Bousmar et al, 2005) in order to get a free surface parallel to the bed and to minimize the net mass exchange between subsections. (b) Two different upstream discharge distributions were investigated for each roughness transition configuration: floodplain discharges $Q_{f}=12$ and $18{\mathrm{~L} . \mathrm{s}^{-1}}^{-1}$ for configuration CWM and $Q_{f}=18$ and $26{\mathrm{~L} . \mathrm{s}^{-1}}^{-}$for configuration CMW. These upstream boundary conditions were chosen to explore different cases of mass transfers between main channel and floodplain in terms of direction and magnitude (see Section 4.1). The levels of the downstream 
weirs were the same as those for the uniform flows over the downstream roughness (e.g. for configuration CMW the downstream weir levels are those of test case CW). This is an arbitrary choice, but justified by the fact that it may facilitate the establishment of the uniform flow state at the flume outlet. The subsection Reynolds number $R e_{i}=4 Q_{i} /\left(\nu\left(2 H_{i}+B_{i}\right)\right)$ ranges from $3.9 \times 10^{4}$ to $9.0 \times 10^{4}$ in the floodplain and from $29.8 \times 10^{4}$ to $42.2 \times 10^{4}$ in the main channel. 


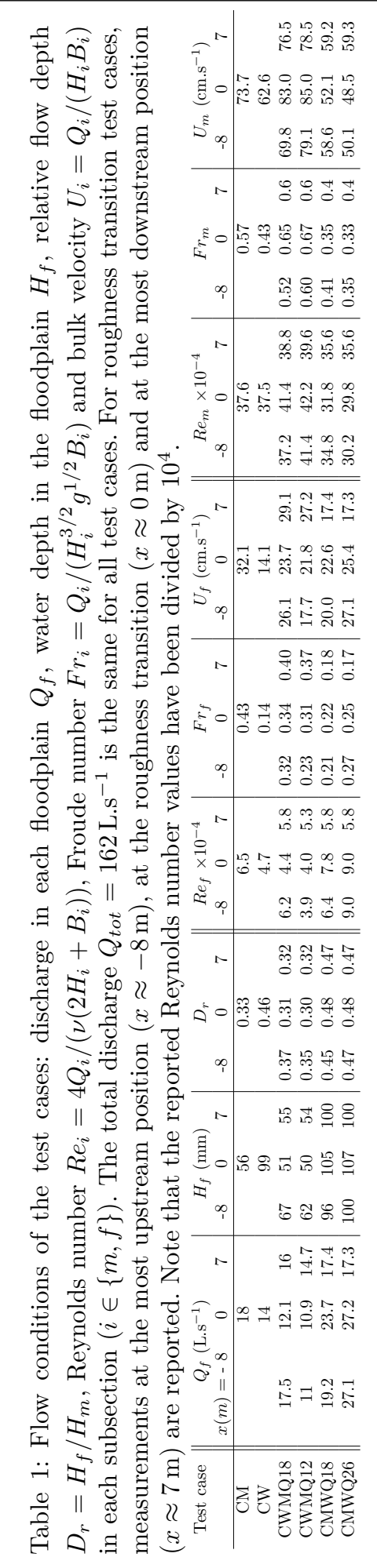


(a)

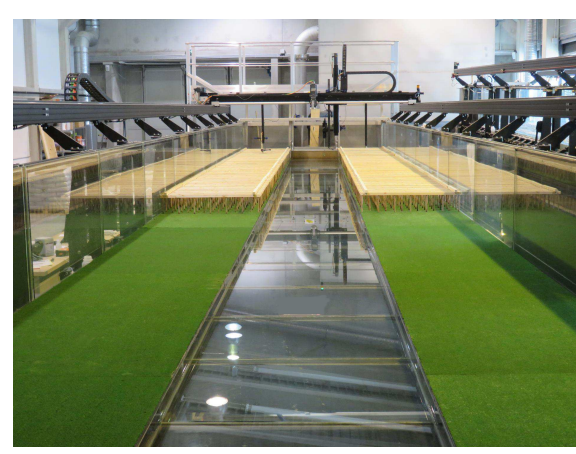

(b)

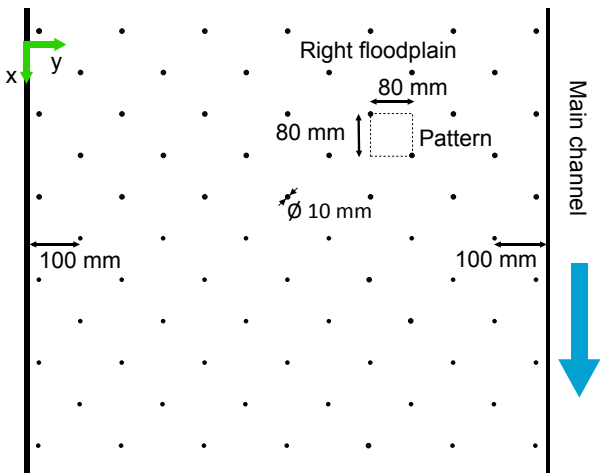

Fig. 2: (a) Compound channel flume at Irstea, longitudinal transition from wood to meadow (configuration CWM, picture viewed from downstream). (b) Plan view of the array of emergent cylinders modelling the wood over the floodplains.

The cylinder array was made of wooden circular cylinders that were uniformly distributed in staggered rows (see Fig. 2b). The cylinder diameter was $D=10 \mathrm{~mm}$ and the cylinder density $N=81$ cylinders. $\mathrm{m}^{-2}$. Cylinders were held together with an emergent wooden superstructure (see Fig. 2a) that does not interact with the flow. The accuracy of the cylinder position was $\pm 5 \mathrm{~mm}$ in both lateral and longitudinal directions.

The free surface elevation was measured with ultrasonic sensors (UNDK20I69, Baumer) with an accuracy of $\pm 0.5 \mathrm{~mm}$. Recording time was $180 \mathrm{~s}$ at a $50 \mathrm{~Hz}$ sampling rate. For the two uniform flows (test cases CM and CW), the water depth was constant for $2 \mathrm{~m}<x_{a}<17 \mathrm{~m}$ in the three subsections with a scatter of $\pm 1 \mathrm{~mm}$. For all flow cases, no significant lateral gradient of free surface level was detected. Velocity was measured by means of a side-looking ADV probe (Vectrino Plus, Nortek). The sampling volume was a $7 \mathrm{~mm}$ long circular cylinder with a $6 \mathrm{~mm}$ diameter. A recording time of $120 \mathrm{~s}$ with a sampling rate of $100 \mathrm{~Hz}$ was sufficient to get converged values of turbulence statistics of first and second orders. For the computation of autocorrelation functions, the recording time was set to $600 \mathrm{~s}$ in order to increase the quality of the results. The ADV raw data were filtered with the free software WinADV, which uses the despiking concept developed by Goring and Nikora (2002).

Owing to cross-section symmetry, we present herein measurements in the right half of the compound section. The vertical plane $(x, z)$ at the main channel/floodplain boundary is called interface, its lateral position is denoted $y_{\text {int }}\left(y_{\text {int }}=1000 \mathrm{~mm}\right.$ for the right-hand interface). In the following, the analysis focuses on the flows subject to a roughness transition (configurations CWM and CMW), using the flows over a uniform roughness (test cases $\mathrm{CM}$ and $\mathrm{CW}$ ) as reference flows.

\section{Longitudinal variation in water depth}

Figure 3 shows the longitudinal profiles of floodplain water depth $H_{f}$ for the roughness transition test cases, along with the mean uniform water depths of test cases 


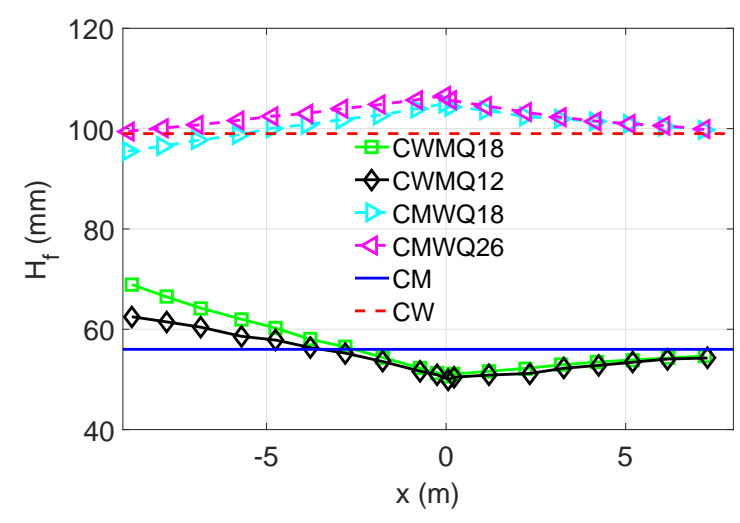

Fig. 3: Longitudinal profiles of floodplain water depth $H_{f}$ for the roughness transition test cases. The uniform water depths (test cases CM and CW) are reminded. Dotted lines refer to test cases ending with wooded floodplains. Measuring position at $y=$ $500 \mathrm{~mm}$.

$\mathrm{CM}$ and CW. As the flow is subcritical along the whole length of the flume, the water surface level is primarily controlled by the downstream weir levels, which are those of the uniform flow over the downstream roughness. For all test cases, an inversion of the free surface slope is observed at the transition. Tests carried out with various downstream weir levels (Dupuis, 2016) revealed that the water depth gradients at the downstream end of the channel are not an artefact due to the downstream weirs. These gradients are intrinsic to the flow dynamics. In an isolated floodplain, the water depth is constant downstream of a change in roughness (Dupuis et al, 2016). It can thus be inferred that the variation in water depth downstream of the roughness transition in a compound channel is related to the lateral mass transfers between subsections, that represent an additional degree of freedom, compared to the single channel (isolated floodplain).

We studied the influence of the upstream discharge distribution between main channel and floodplain on the $H_{f}(x)$-profile in varying the value of $Q_{f}$ at the flume entrance for a given configuration, $Q_{t o t}=Q_{m}+2 Q_{f}$ being kept constant. Test case CWMQ18 is first taken as a reference flow. The difference in water depth compared to this reference flow, $H_{f}-H_{f, C W M Q 18}$ is shown in Fig. 4a. The same tests were carried out with test case CMWQ18 as reference flow (Fig. 4b). For the two roughness transitions, an increase in floodplain inflow induces an increase in water depth, for the same total discharge. For the wood-to-meadow transition (Fig. 4a), the upstream discharge distribution has an effect mainly upstream of the transition. For the meadow-to-wood transition (Fig. 4b), the discrepancy of $H_{f}$ with the reference flow is nearly proportional to the downstream distance. Note that for the meadowto-wood transition, a local singularity of $H_{f}$ is observed at the transition (Fig. 4b), which is due to a stationary wave at this location.

We can therefore conclude that the water depth is primarily controlled by the downstream boundary condition, like roughness transitions in a single channel, and secondarily controlled by the upstream boundary condition (discharge distribution). This latter control is due to the at least two-dimensional character of the flow, which 

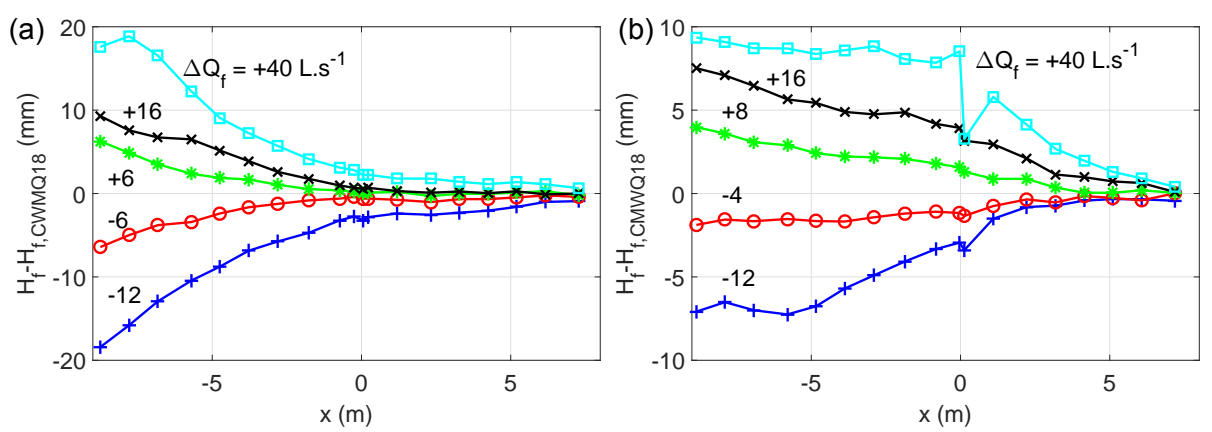

Fig. 4: (a) Longitudinal profiles of floodplain water depth $H_{f}$ relative to the water depth profile of test case CWMQ18 $\left(H_{f, C W M Q 18}\right)$ for the wood-to-meadow transition for different upstream discharge distributions. (b) Longitudinal profiles of floodplain water depth relative to the water depth profile of test case CMWQ18 $\left(H_{f, C M W Q 18}\right)$ for the meadow-to-wood transition for different upstream discharge distributions. The variation in floodplain discharge (in L.s ${ }^{-1}$ ) relative to the reference test case $\left(Q_{f}=18 \mathrm{~L} . \mathrm{s}^{-1}\right)$ is denoted $\Delta Q_{f}$. Measuring position at $y=500 \mathrm{~mm}$.

is linked to the lateral mass transfers between subsections, compared to the $1 \mathrm{D}$ single channel flow.

\section{Lateral mass transfers}

\subsection{Discharge distribution between subsections}

The velocity field was measured in the right-hand half cross-section of the main channel at nine $x$-stations for each roughness transition test case. The measuring mesh had a mean lateral spacing of $50 \mathrm{~mm}$ and a mean vertical spacing of $8 \mathrm{~mm}$ (about 250 measuring points in the half cross-section). The mesh was refined near the interface. Assuming symmetry of the flow, the discharge distribution between subsections was then calculated by integration of the velocity field. The longitudinal variation in floodplain discharge $Q_{f}(x)$ is shown in Fig. 5. The upstream discharge distributions were chosen such that, for each transition type, there was one test case with lateral mass exchanges upstream of the roughness transition (CWMQ18 and CMWQ18) and one test case without lateral mass exchanges upstream of the transition (CWMQ12 and CMWQ26). Downstream of the roughness transition, mass transfers are always present and are in the same direction for the two test cases of each transition type. The direction of the mass transfers between main channel and floodplain is reported in Table 2.

Downstream of the transition, the discharge distribution tends towards the distribution of the uniform flow with the downstream roughness (horizontal lines), but the latter is not reached. According to Rominger and Nepf (2011), the flow adjustment length downstream of the leading edge of a cylinder array set in the middle of a flat channel is $L_{d w} \approx 6\left(1+\left(C_{D} a b\right)^{2}\right) /\left(C_{D} a\right)$, where $b$ is the half width of the cylinder array, $C_{D}$ is the cylinder drag coefficient and $a$ is the frontal area per unit volume. 
Table 2: Directions of lateral mass transfers upstream $(x<0)$ and downstream $(x>0)$ of the roughness transition. $\mathrm{MC}=$ main channel. $\mathrm{FP}=$ floodplain. $\varnothing=$ no transfer.

\begin{tabular}{lcccc}
\hline & CWMQ18 & CWMQ12 & CMWQ18 & CMWQ26 \\
\hline$x<0$ & $\mathrm{FP} \rightarrow \mathrm{MC}$ & $\varnothing$ & $\mathrm{MC} \rightarrow \mathrm{FP}$ & $\varnothing$ \\
$x>0$ & $\mathrm{MC} \rightarrow \mathrm{FP}$ & $\mathrm{MC} \rightarrow \mathrm{FP}$ & $\mathrm{FP} \rightarrow \mathrm{MC}$ & $\mathrm{FP} \rightarrow \mathrm{MC}$ \\
\hline
\end{tabular}

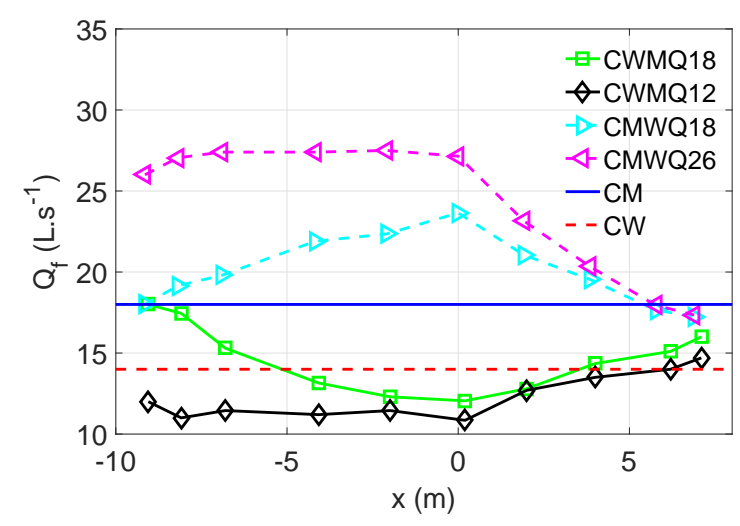

Fig. 5: Longitudinal variation in floodplain discharge $Q_{f}$ for the four test cases with a roughness transition; the floodplain discharge of the uniform meadow $(\mathrm{CM})$ and uniform wood (CW) test cases are reminded with blue and red horizontal lines, respectively. Dashed lines refer to test cases ending with wooded floodplains.

This distance is defined in their study as the downstream position along the centreline of the cylinder array where the longitudinal velocity becomes constant. It should therefore fairly correspond to the distance where lateral mass transfers become zero. Applied to the present meadow-to-wood transition, with $b$ the floodplain width and taking $C_{D}=1.2$ (Dupuis et al, 2016) and $a=N D=0.81 \mathrm{~m}^{-1}$, this formula gives $L_{d w}=3.2 \mathrm{~m}$. However, for configuration CMW, mass transfers are still present $7 \mathrm{~m}$ downstream of the roughness change. Mass exchanges thus appeared to be slower in a compound channel than in a flat channel. This fact can be explained by considering the vertical surface available for the lateral mass exchange, which spans the whole water column for the flat channel, but is reduced to the floodplain flow depth for the compound channel flow.

4.2 Effect of lateral mass transfers in the momentum balance of each subsection

We show in the Appendix that the normalised total force related to the lateral mass transfers either on the main channel flow $(i=m)$ or on the floodplain flow $(i=f)$ is:

$$
A_{i}=\frac{2 U_{i}-U_{d, i n t}}{g H_{i}} \frac{\partial H_{i} U_{i}}{\partial x}
$$


where $H_{i}$ is the subsection water depth, $U_{i}$ is the subsection bulk velocity and $U_{d, i n t}$ is the depth-averaged longitudinal velocity at the interface. Term $A_{i}$ can be split up into two contributions: (1) the acceleration due to flow contraction when fluid enters the subsection (or the deceleration due to flow expansion when fluid leaves the subsection):

$$
A_{c, i}=\frac{U_{i}}{g H_{i}} \frac{\partial H_{i} U_{i}}{\partial x}
$$

and (2) the acceleration/deceleration due to the difference between the velocity of the entering/leaving fluid and the subsection-averaged velocity:

$$
A_{a, i}=\frac{U_{i}-U_{d, i n t}}{g H_{i}} \frac{\partial H_{i} U_{i}}{\partial x}
$$

When the velocity of the entering flow $U_{d, i n t}$ is equal to the mean velocity in the subsection $U_{i}$, then $A_{i}=A_{c, i}$.

Considering that $U_{f} \leqslant U_{d, i n t} \leqslant U_{m}$, the term $2 U_{m}-U_{d, \text { int }}$ is positive. Therefore a mass gain $\left(\frac{\partial H_{m} U_{m}}{\partial x}>0\right)$ induces a resistance force in the main channel $\left(A_{m}>0\right)$ and a mass loss $\left(\frac{\partial H_{m} U_{m}}{\partial x}<0\right)$ induces a driving force $\left(A_{m}<0\right)$. For a given mass transfer direction, the two contributions $A_{c, m}$ and $A_{a, m}$ are of the same sign.

In the floodplain, two cases have to be distinguished: (1) if $2 U_{f}-U_{d, \text { int }} \geqslant 0$, then the mass transfer effect is the same as discussed above for the main channel; (2) if $2 U_{f}-U_{d, \text { int }}<0$, a mass gain induces a driving force $\left(A_{f}<0\right)$ and a mass loss induces a resistance force $\left(A_{f}>0\right)$. In both cases, as $U_{f} \leqslant U_{d, i n t}, A_{c, f}$ and $A_{a, f}$ are of opposite sign in the floodplain and the two contributions partly cancel each other.

Figure 6 shows the ratios $A_{c, i} / S_{0}, A_{a, i} / S_{0}$ and $A_{i} / S_{0}$, which represent the two contributions of the total force induced by net lateral mass exchange, together with the sum of these two contributions, normalised by the gravitational force. The two forces $A_{c, i}$ and $A_{a, i}$ are of the same order of magnitude. As stated above, they add each other in the main channel, which results in an important net force $A_{m}$ that is up to twice the gravitational force. By contrast, the two contributions almost cancel each other out in the floodplain, resulting in a very small force $A_{f}$. For all test cases investigated we are in the case (1) $2 U_{f}-U_{d, \text { int }} \geqslant 0$, such that force $A_{f}$ is resistant when mass enters the floodplain.

\section{Mixing layer dynamics}

The difference in velocity between the flows in the deeper main channel and in the shallower floodplain generates a mixing layer at the interface. This mixing layer is investigated in the present section at the constant altitude $z_{f}=H_{f} / 2$. Lateral profiles of mean velocities and turbulence quantities have been measured at different $x$-stations for each transition. As shown in our previous study (Dupuis et al, 2017), the properties of the compound channel mixing layer (width, dimensionless velocity difference $\lambda$, etc.) change across the water column. The vertical position of the measurements only varies between $\pm 3 \mathrm{~mm}\left( \pm 5 \%\right.$ of $\left.H_{f}\right)$ along the flume for a given test case. Therefore, the longitudinal variations that are observed for a given test case can be attributed to the longitudinal flow development and not to the effect of the $z$-variation of the measuring point. 

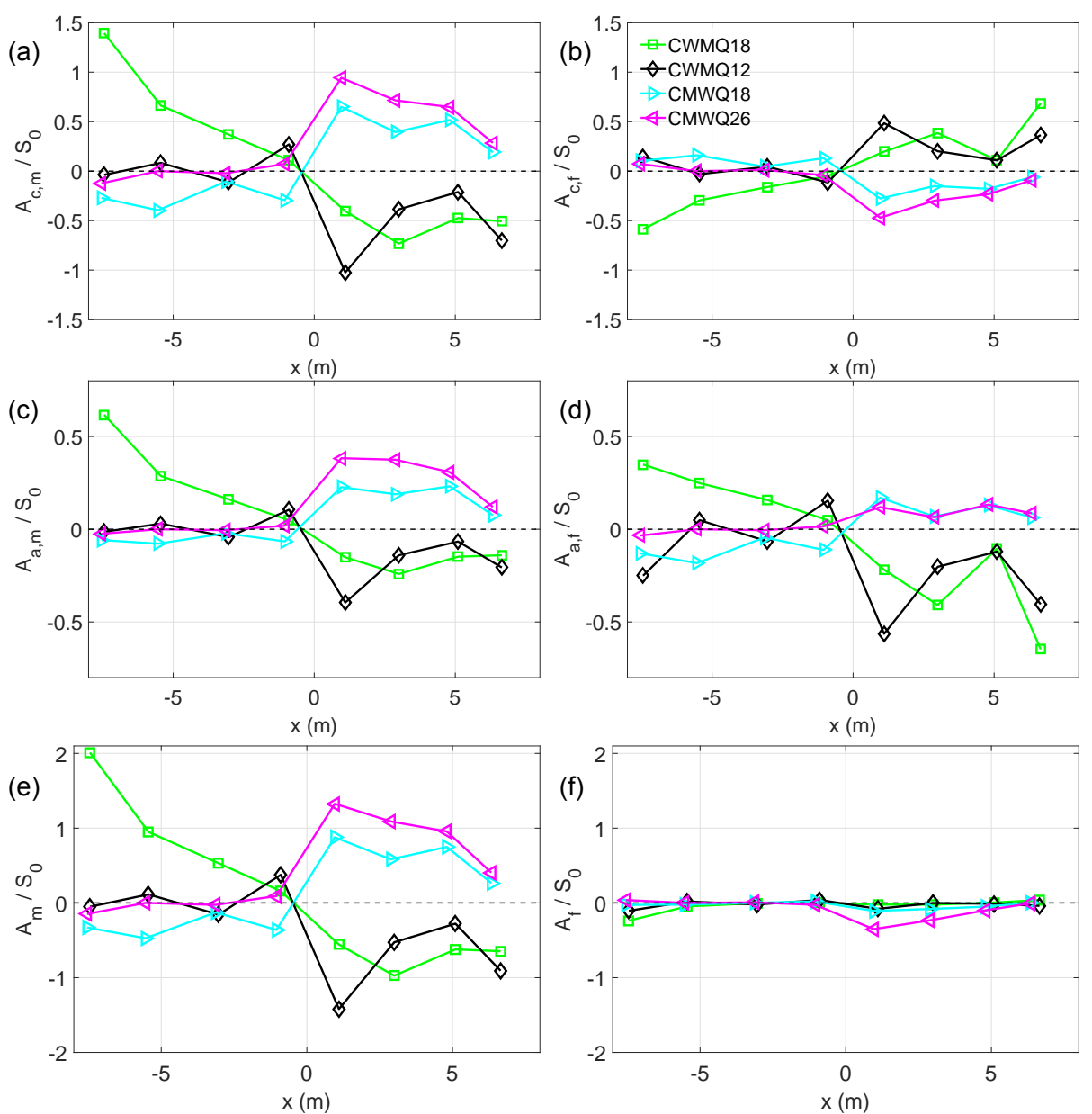

Fig. 6: (a-b) Compression force due to lateral net mass exchanges (Eq. 2), (c-d) acceleration due to mass exchanges (Eq.3), (e-f) net force due to mass exchanges (Eq. 1) in the main channel (left panels) and in the floodplain (right panels).

\subsection{Mixing layer centre}

Figure 7 shows an example of a profile $U(y)$ measured across the mixing layer for test case CMWQ18 at $x=-2.5 \mathrm{~m}$ and $z_{f}=H_{f} / 2$. Contrary to the plane mixing layer that features an antisymmetric velocity profile, here the inflection point position $y_{I P}$ does not collapse with the geometrical centre of the mixing layer, nor with the position $y_{U_{0}}$ where the velocity $U_{0}=\left(U_{1}+U_{2}\right) / 2$ is reached, with $U_{1}$ the maximum velocity in the main channel and $U_{2}$ the velocity in the floodplain outside the mixing layer in the plateau region. Since the inflection point is the primary source of flow instability (Fjortoft, 1950), we define it as the centre of the mixing layer.

The position of the inflection point is quasi invariant in the longitudinal direction for all test cases investigated, with $y_{I P}=y_{i n t} \pm 10 \mathrm{~mm}$. The position of $y_{I P}$ is 


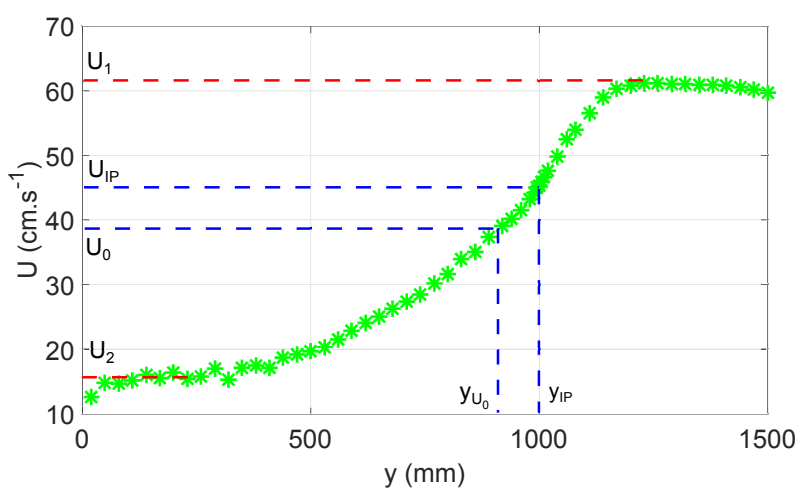

Fig. 7: Lateral profile of longitudinal mean velocity $U(y)$ at $z_{f}=H_{f} / 2$ and $x=-2.5 \mathrm{~m}$ for test case CMWQ18. Maximum velocity $U_{1}$, plateau velocity in the floodplain $U_{2}$, averaged velocity $U_{0}=\left(U_{1}+U_{2}\right) / 2$, inflection point position $y_{I P}$ and position of $y_{U_{0}}$ are indicated.

therefore essentially controlled by the location of the change in flow depth. Unlike Proust et al (2013) and Peltier et al (2013), we do not detect a lateral displacement of $y_{I P}$ when mass is transferred from the floodplain to the main channel. This may be due to weaker mass transfers than in the experiments of Proust et al (2013) and Peltier et al (2013).

Figure $8 \mathrm{a}$ shows the position $y_{U_{0}}$ relative to the position $y_{i n t}$ for all test cases. For the two flows without transition in roughness $(\mathrm{CM}$ and $\mathrm{CW})$, position $y_{U_{0}}$ slightly moves towards the main channel when going downstream. For the flows with a transition in roughness, position $y_{U_{0}}$ much more varies and can be related to the lateral mass exchange between floodplain and main channel. The magnitude of the lateral mass transfers is shown in Fig. 8b, quantified by the depth-averaged mean lateral velocity at the interface $V_{d, \text { int }}$, calculated from the longitudinal variation of floodplain discharge $Q_{f}$. When mass is transferred from the floodplain to the main channel (upstream of the transition for test case CWMQ18 and downstream of the transition for test cases CMWQ18 and CMWQ26), $y_{U_{0}}$ is displaced towards the main channel and $y_{U_{0}}-y_{\text {int }}>0$ (right-hand interface). On the contrary, when mass is transferred from the main channel to the floodplain (downstream of the transition for test cases CWMQ18 and CWMQ12 and upstream of the transition for test case CMWQ18), $y_{U_{0}}$ is displaced towards the floodplain. However, the comparison of Figs. 8a and 8b shows that the displacement of $y_{U_{0}}$ is not proportional to the magnitude of the transfers, e.g. in the upstream reach of CMWQ18, the displacement of $y_{U_{0}}$ is the highest although the lateral mean velocity is low. Indeed, the displacement of $y_{U_{0}}$ is also sensitive to the lateral gradient of $U(y)$ in the mixing layer region. For high lateral gradients of $U(y), y_{U_{0}}-y_{\text {int }}$ necessarily varies over a shorter range than for low gradients. For example, $U(y)$ has a much lower gradient for test case CMWQ18 at $y=-5 \mathrm{~m}$ than for test case CWMQ18 at the same position (not shown). Therefore, $y_{U_{0}}$ moves much strongly in the former case. 

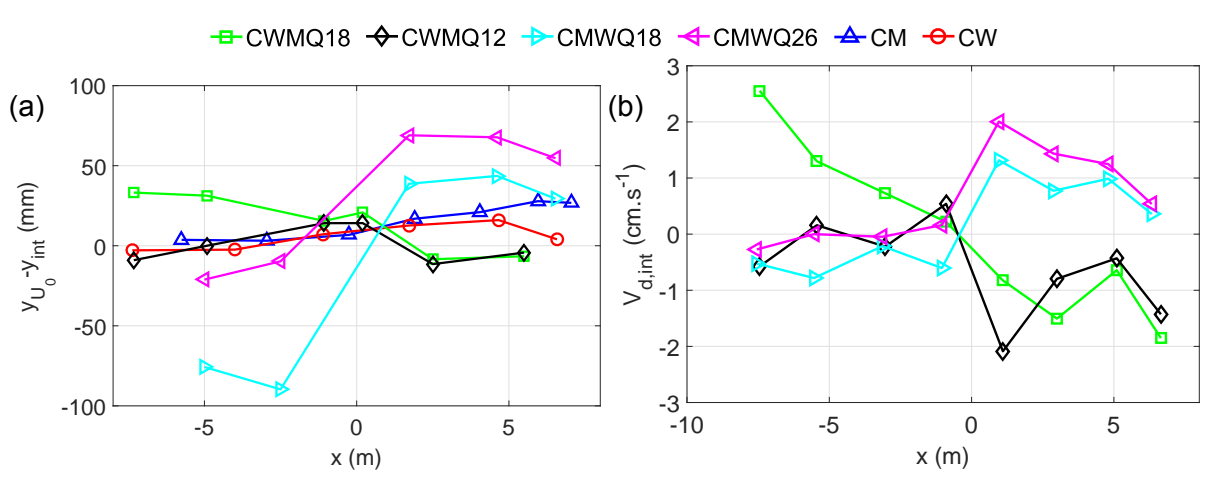

Fig. 8: (a) Longitudinal evolution at $z_{f}=H_{f} / 2$ of the lateral position $y_{U_{0}}$, where the velocity $U_{0}$ is reached. (b) Longitudinal evolution of the mean lateral depth-averaged velocity at the interface $V_{d, i n t}$.

\subsection{Mixing layer width}

To take into account the asymmetry of the compound channel mixing layer with respect to the interface, two mixing layer widths are defined on either side of the interface: the main channel mixing layer width $\delta_{m}$ and the floodplain mixing layer width $\delta_{f}$ :

$$
\begin{aligned}
& U\left(y_{i n t}+\delta_{m} / 2\right)=\frac{U_{1}+U_{i n t}}{2} \\
& U\left(y_{i n t}-\delta_{f} / 2\right)=\frac{U_{2}+U_{i n t}}{2}
\end{aligned}
$$

where $U_{\text {int }}$ is the velocity at the interface. In Eq. 4 and 5 and thereafter we consider that $y_{\text {int }}$ coincides with the position of the inflection point $y_{I P}$.

Figure 9 shows the longitudinal evolution of $\delta_{m}$ and $\delta_{f}$ for the various test cases. For the two test cases with uniform roughness $(\mathrm{CM}$ and $\mathrm{CW}), \delta_{m}$ grows until the channel outlet while $\delta_{f}$ levels off at $x \approx 4 \mathrm{~m}$ and $x \approx-4 \mathrm{~m}$ for test cases CM and CW, respectively. With a roughness transition, the value $\delta_{f}$ undergoes important changes at the transition: the floodplain-side of the mixing layer is widening downstream of the wood-to-meadow transition and is narrowing downstream of the meadow-towood transition. Width $\delta_{m}$ is less affected by the longitudinal change in roughness.

Plane mixing layer studies (Brown and Roshko, 1974) showed that the mixing layer width increases with an increasing normalised velocity difference $\lambda=$ $\left(U_{1}-U_{2}\right) /\left(U_{1}+U_{2}\right)$. It is also known from shallow mixing layer studies that flow confinement and bed roughness tend to constraint the lateral extent of the mixing layer (a decrease in $H$ or an increase in bed roughness lead to a decrease in $\delta$ ). Chu and Babarutsi (1988) suggested to normalise the shallow mixing layer width $\delta$ in the way $\delta^{*}=\delta c_{f} /(H \lambda)$ where $c_{f}$ is the averaged bed friction coefficient across the mixing layer $\left(c_{f}=0.5\left(c_{f 1}+c_{f 2}\right)\right.$ with $c_{f i}=\tau_{b} /\left(0.5 \rho U_{i}^{2}\right), i \in\{1,2\}$ and $\tau_{b}$ the bed shear stress) and showed that for flows that are shallow enough the quantity $\delta^{*}$ converges towards a constant value when going downstream $\left(\delta_{\text {max }}^{*} \approx 0.13\right)$. In the following a similar normalisation of the mixing layer width as done by $\mathrm{Chu}$ and Babarutsi (1988) is carried out. 

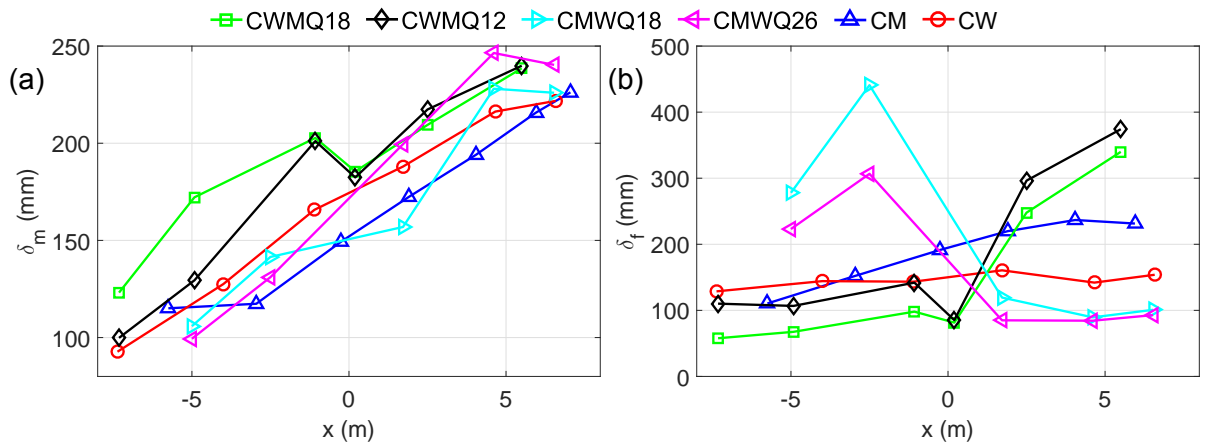

Fig. 9: Longitudinal evolution of (a) main channel mixing layer width $\delta_{m}$ and (b) floodplain mixing layer width $\delta_{f}$ at $z_{f}=H_{f} / 2$.

To take into account the asymmetrical character of the compound channel mixing layer, specific $\lambda$-values are defined in the main channel and in the floodplain:

$$
\begin{aligned}
& \lambda_{m}=\frac{U_{1}-U_{i n t}}{U_{i n t}} \\
& \lambda_{f}=\frac{U_{i n t}-U_{2}}{U_{i n t}} .
\end{aligned}
$$

For an antisymmetric mixing layer $\lambda_{m}=\lambda_{f}=\lambda$.

The main channel and floodplain mixing layer widths are then normalised in the way:

$$
\begin{aligned}
& \delta_{m}^{+}=\frac{\delta_{m}}{\lambda_{m} H_{f}} \\
& \delta_{f}^{+}=\frac{\delta_{f}}{\lambda_{f} H_{f}} .
\end{aligned}
$$

Note that $H_{f}$ and not $H_{m}$ is used to normalise $\delta_{m}$, since $H_{f}$ is the vertical extension of the mixing layer. According to Chu and Babarutsi (1988) $\delta^{*}$ should be a constant and thus $\delta_{m}^{+}$and $\delta_{f}^{+}$should only depend on the bed friction coefficient $\left(\delta^{+}=\delta^{*} c_{f}\right)$. In the present experiments, the bed friction coefficient $c_{f}$ is not adapted for describing emergent roughness elements.

Figure 10 shows the longitudinal evolution of $\delta_{m}^{+}$and $\delta_{f}^{+}$. Both normalised widths $\delta_{m}^{+}$and $\delta_{f}^{+}$are equal and rather constant along the channel for test case CW with a mean value of 2 . For test case $\mathrm{CM}, \delta_{m}^{+}$increases all along the flume while $\delta_{f}^{+}$levels off at $x \approx 4 \mathrm{~m}$, the values being higher than for test case CW, i.e. between 4 and 9 . For the test cases with a roughness transition, we observe that $\delta_{m}^{+}$and $\delta_{f}^{+}$approximately follow the trend of the uniform flow test cases (CM or $\mathrm{CW}$ ) corresponding to the floodplain roughness in the reach considered. One exception is the upstream reach of the wood-to-meadow transitions (test cases CWMQ18 and CWMQ12), where $\delta_{m}^{+}$is about twice higher than the value related to the uniform flow test case CW (Fig. 10a). This suggests that the proposed normalisation $\delta_{i}^{+}$has its limitations and do not take into account all physical processes that govern $\delta_{i}$. 

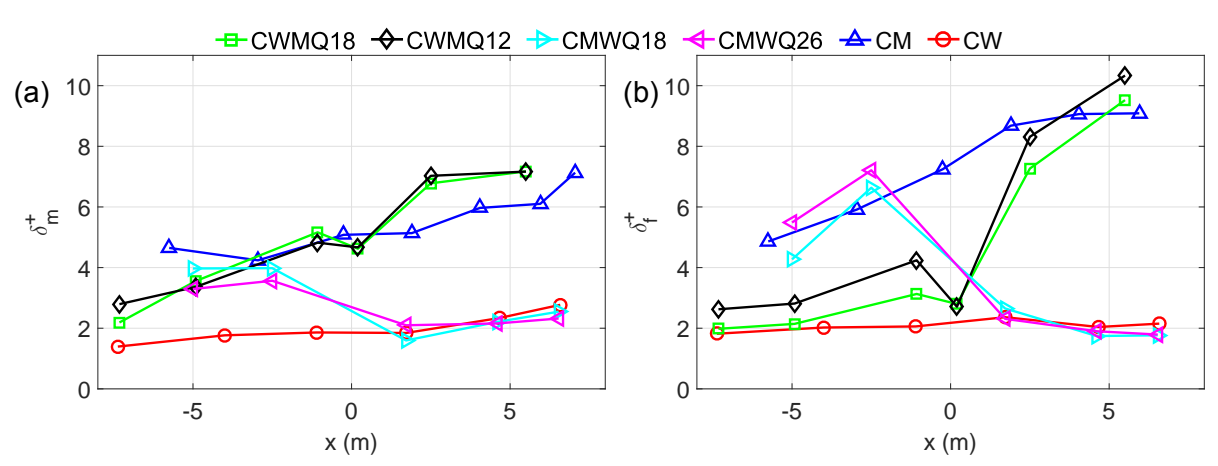

Fig. 10: (a) Main channel and (b) floodplain mixing layer widths at $z_{f}=H_{f} / 2$, normalised by $\lambda_{i}$ and floodplain water depth (Eqs. 8 and 9).

The larger normalised mixing layer widths for reaches with grassed floodplains than with wooded floodplains (about three times higher) can be related to the obstruction caused by the cylinder array, which limits the lateral penetration of the coherent structures into the floodplain (White and Nepf, 2007).

\subsection{Normalised lateral profiles}

In our previous study (Dupuis et al, 2017), we observed that for the two flows with uniform roughness $\mathrm{CM}$ and $\mathrm{CW}$, the normalised profiles of longitudinal velocity and turbulence quantities were self-similar when going downstream. The velocity was normalised in the form $\left(U-U_{2}\right) /\left(U_{1}-U_{2}\right)$ and, to take into account the mixing layer asymmetry, the lateral coordinate was normalised by the subsection mixing layer width, i.e. by $\delta_{m}$ on the main channel side and by $\delta_{f}$ on the floodplain side. For the roughness transition test cases, the lateral position $y_{U_{0}}$ where the velocity $U_{0}=\left(U_{1}+U_{2}\right) / 2$ is reached can be far away from the mixing layer centre $y_{\text {int }}$ (Fig. 8). Therefore the lateral velocity profiles are not self-similar when normalised in the form $\left(U-U_{2}\right) /\left(U_{1}-U_{2}\right)$; indeed, the profiles do not pass necessarily through the point $(0,0)$ any more (Dupuis, 2016). To take into account the mixing layer asymmetry due to the displacement of $y_{U_{0}}$, the velocity profiles are normalised in Fig. 11 in the form:

$$
\frac{U-U_{i n t}}{\left|U_{i}-U_{i n t}\right|}=f\left(\frac{y-y_{i n t}}{\delta_{i}}\right)
$$

where $U_{i}=U_{1}$ and $\delta_{i}=\delta_{m}$ on the main channel side $\left(y>y_{\text {int }}\right.$ at the right-hand interface) and $U_{i}=U_{2}$ and $\delta_{i}=\delta_{f}$ on the floodplain side $\left(y<y_{\text {int }}\right)$.

Figure 11a shows normalised profiles of $U(y)$ in reaches with grassed floodplains, downstream of the transition for test case CWMQ18 and upstream of the transition for test case CMWQ26, along with the self-similar profile of test case CM. Similarly, Fig. 11b shows normalised profiles of $U(y)$ in reaches with wooded floodplains, upstream of the transition for test case CWMQ12 and downstream of the transition for test case CMWQ18, together with the self-similar profile of test case CW. In the 

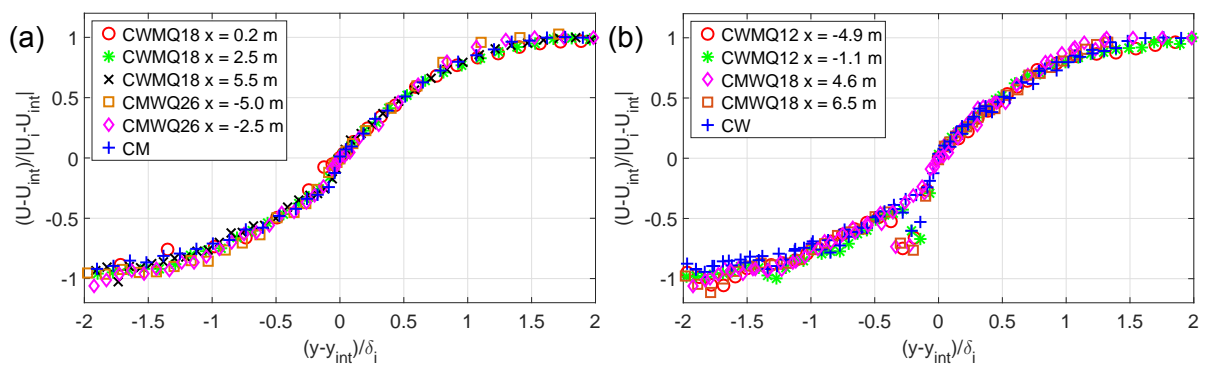

Fig. 11: Lateral normalised profiles (after Eq.10) of mean longitudinal velocity at $z_{f}=H_{f} / 2$ and at various $x$-stations (a) with grassed floodplains for test cases CWMQ18 and CMWQ26 and (b) with wooded floodplains for test cases CWMQ12 and CMWQ18.

frame of the normalisation given by Eq. 10, all $U(y)$-profiles coincide, and the profiles in grassed and wooded reaches also match together (the only difference is due to the cylinder wakes that are only present in the wooded reaches). We can thus conclude that this velocity profile shape is independent of (i) water depth, (ii) normalised velocity difference $\lambda_{i}$, (iii) averaged velocity $U_{0}$, (iv) lateral net mass exchange between subsections and (v) floodplain roughness. In other words, all modifications on the $U(y)$-profile induced by variations in these five parameters are taken into account in Eq. 10. Note that the difference in the cylinder wake location in Fig. 11b is due to the scaling of the lateral coordinate by $\delta_{f}$ (although $\delta_{f}$ varies, the lateral position of the cylinder is fixed).

Figure 12 shows the profiles of lateral Reynolds stress $\rho \overline{u^{\prime} v^{\prime}}(y)$ at the same $x$ stations than in Fig 11, normalised in the form $-\overline{u^{\prime} v^{\prime}} /\left(U_{i}-U_{i n t}\right)^{2}=f\left(\left(y-y_{i n t}\right) / \delta_{i}\right)$, consistently with Eq. 10. These profiles do not collapse. Therefore the flows are not fully self-similar, but only partially self-similar in the sense of George (1989), i.e. only for the mean flow but not for turbulence moments of higher orders. The contrast between the superposition of the mean velocity profiles and the diversity of the turbulence quantity profiles was already pointed out by Townsend (1961) in the case of wall flows. The non-univocity between velocity and shear stress profiles implies in particular that the eddy viscosity model is not valid. The turbulence is not only due to local production by mean velocity gradients, but is driven by a complex physics where come into play coherent structures and flow history. Tests with other normalisations of the lateral shear stress (e.g. $\left.-\overline{u^{\prime} v^{\prime}} /\left(U_{1}-U_{2}\right)^{2}\right)$ show no superposition of the profiles too.

\section{Coherent structures}

The time series $u(t)$ and $v(t)$ feature large quasi periodic oscillations in the interface region. These oscillations are the signature of coherent structures that are generated by the mixing layer. 

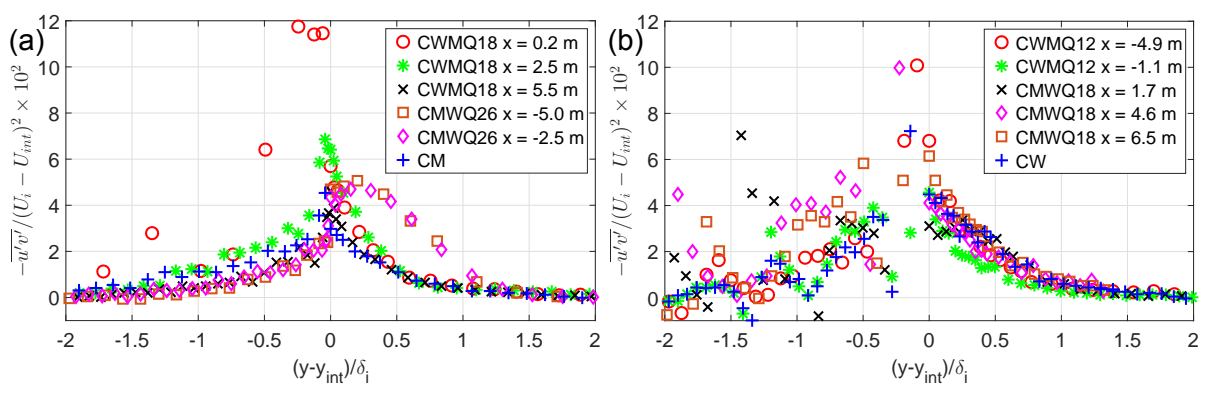

Fig. 12: Lateral normalised profiles of lateral Reynolds stress at $z_{f}=H_{f} / 2$ and at various $x$-stations (a) with grassed floodplains for test cases CWMQ18 and CMWQ26 and (b) with wooded floodplains for test cases CWMQ12 and CMWQ18.

\subsection{Longitudinal length scale}

The Eulerian integral time scale $\tau_{i i}$ of the longitudinal $(i=1)$ or lateral $(i=2)$ velocity fluctuations can be calculated as four times the first zero-crossing of the autocorrelation function of the velocity signal, since the first zero crossing corresponds to the quarter of period for a periodic signal. The longitudinal Eulerian integral length scales $L_{i i}^{(1)}$ can then be calculated with the Taylor hypothesis using the relation $L_{i i}^{(1)}=U_{d, i n t} \tau_{i i}$, where $U_{d, i n t}$ is the depth-averaged mean longitudinal velocity at the interface. We showed in Dupuis et al (2017) that this velocity is a fair estimate of the convection velocity of the coherent structures. Length scales $L_{11}^{(1)}$ and $L_{22}^{(1)}$ characterise the longitudinal size of the coherent structures considering the longitudinal and lateral velocity fluctuations.

Figure 13 shows the longitudinal evolution of length scales $L_{11}^{(1)}$ and $L_{22}^{(1)}$ at the interface and at $z_{f}=H_{f} / 2$. The coherent structures continuously grow when going downstream for test case $\mathrm{CM}$ with uniform grassed floodplains. By contrast, the coherent structure length is rather constant after $x \approx 2 \mathrm{~m}$ for test case CW with uniform wooded floodplains. A change in trend is observed downstream of the roughness transition, for test cases with a change in roughness. An increase in the growth rate is observed downstream of the wood-to-meadow transition. On the other hand, the meadow-to-wood transition is characterised by a decrease in the coherent structure length, followed by a new increase after $x \approx 2-3 \mathrm{~m}$. The evolution trends are the same for $L_{11}^{(1)}$ and $L_{22}^{(1)}$ but $L_{11}^{(1)}$ is systematically higher than $L_{22}^{(1)}$ : the ratio $L_{11}^{(1)} / L_{22}^{(1)}$ is in average equal to 1.3 . This value is very close to those measured by Nikora et al (2007) in the case of large-scale coherent structures in super- and subcritical shallow open-channel flows $\left(L_{11}^{(1)} / L_{22}^{(1)}=1.19\right.$ to 1.37$)$.

Figure 14 shows the ratios $L_{i i}^{(1)} / \delta_{\text {tot }}$, where $\delta_{t o t}=\delta_{m}+\delta_{f}$ is the total mixing layer width. Only $x$-stations where $\delta_{\text {tot }}$ is available are plotted. Although an important scatter is observed between the different flow cases, it appears that the length scales $L_{i i}^{(1)}$ approximately scale with $\delta_{t o t}$. Moreover, the ratios $L_{i i}^{(1)} / \delta_{t o t}$ are not dependent on the roughness type (meadow or wood). The mean values between all flow cases are $L_{11}^{(1)} / \delta_{\text {tot }}=5.5$ and $L_{22}^{(1)} / \delta_{\text {tot }}=4.2$. 

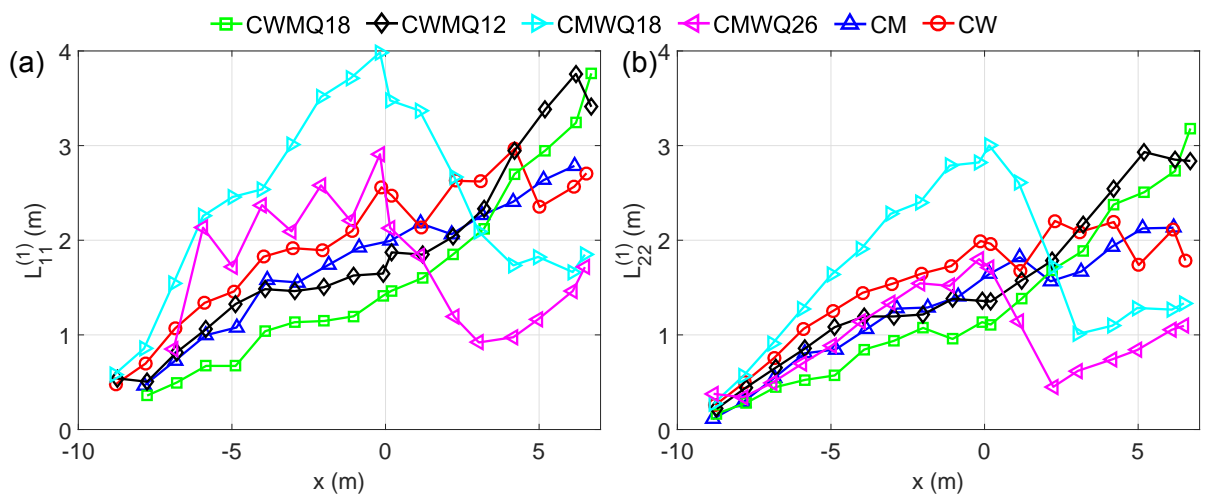

Fig. 13: Longitudinal evolution of the longitudinal integral length scales of (a) the longitudinal velocity and (b) the lateral velocity, at the interface and at $z_{f}=H_{f} / 2$.
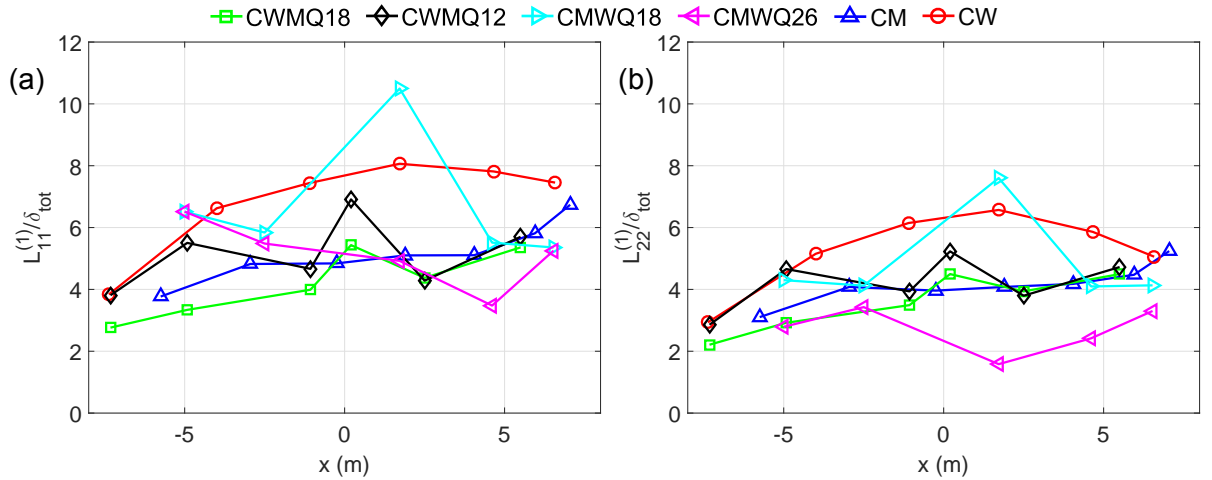

Fig. 14: Longitudinal evolution of (a) ratio $L_{11}^{(1)} / \delta_{\text {tot }}$ and (b) ratio $L_{22}^{(1)} / \delta_{\text {tot }}$.

Knowing now that $L_{i i}^{(1)}$ is proportional to $\delta_{t o t}=\delta_{m}+\delta_{f}$, the decrease in $L_{i i}^{(1)}$ downstream of the meadow-to-wood transition can be related to the decrease in $\delta_{f}$ in this region (Fig. 9b) and the following increase in $L_{i i}^{(1)}$ after $x \approx 2-3 \mathrm{~m}$ to the increase in $\delta_{m}$ (Fig. 9a).

\subsection{Process of growth}

The velocity fluctuations at the interface are primarily due to the large-scale vortices with vertical axes. Assuming a sinusoidal shape of the fluctuations, we can relate the amplitude of the fluctuations of $u^{\prime}$ to $\sqrt{\overline{u^{\prime 2}}}$. Figure 15 shows the longitudinal evolution of the depth-averaged velocity fluctuations at the interface $\sqrt{\overline{u^{\prime 2}}}$,int and $\sqrt{\overline{v^{\prime 2}}} d$, int . For all roughness transition test cases, the amplitude of the oscillations is either constant (CMW) or increasing (CWM) upstream of the transition, while the coherent structures are growing (Fig. 13). By contrast, amplitude of the oscillations 

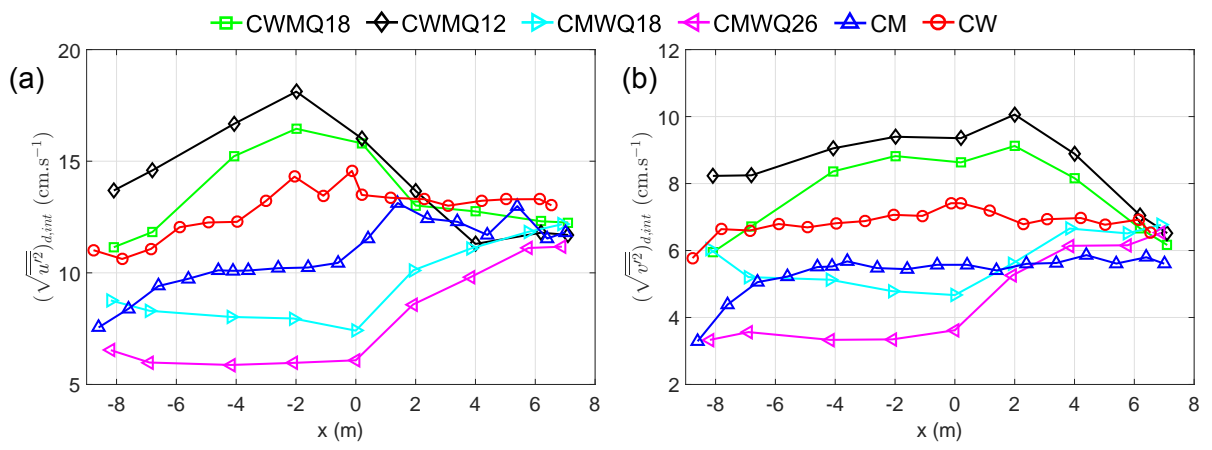

Fig. 15: Longitudinal evolution in the amplitude of the (a) longitudinal and (b) lateral velocity fluctuations, averaged over the depth at the interface.

and coherent structure size evolve in an opposite way immediately downstream of the transition, e.g. for the meadow-to-wood transition, the amplitude of the oscillations increases while the coherent structures are shrinking. We propose the following qualitative explanation.

If $R_{c}$ is the typical radius of a vortex structure and $\omega$ its rotation speed, the vortex structure angular momentum is proportional to $\omega R_{c}^{2}$. Qualitatively we can assume the scaling relations $R_{c} \sim L_{22}^{(1)}$ and $\omega R_{c} \sim \sqrt{\overline{v^{\prime 2}}}$, and therefore that angular momentum is proportional to $L_{22}^{(1)} \sqrt{\overline{v^{\prime 2}}}$. For the two uniform flows CM and CW and in the region upstream of the transition for flows CWM and CMW, $L_{22}^{(1)}$ continuously increases while $\sqrt{\overline{v^{\prime 2}}}$ is constant or increases too. Therefore angular momentum is not conserved and we can infer that the coherent structure growth is associated with structure interaction like vortex merging (Winant and Browand, 1974) and/or fluid entrainment (Moore and Saffman, 1975), as for plane mixing layers. Immediately downstream of the change in roughness, the variations in $L_{22}^{(1)}$ and $\sqrt{\overline{v^{\prime 2}}}$ are opposite, such that the variation of $L_{22}^{(1)} \sqrt{\overline{v^{\prime 2}}}$ is limited. This suggests that in these regions the coherent structures are mostly driven by a process conserving angular momentum, like vortex stretching.

\subsection{Equilibrium}

Figure 16 shows the same quantities as in Fig. 15, normalised by the depthaveraged longitudinal mean velocity at the interface $U_{d, i n t}$, which is also considered as the convection velocity of the coherent structures. It appears that the amplitude of the coherent oscillations scales with $U_{d, i n t}$ for a given floodplain roughness. The normalised turbulence intensities are higher for the wooded floodplains $\left[\left(\sqrt{\overline{u^{\prime 2}}}\right)_{d, \text { int }} / U_{d, \text { int }} \approx 0.35\right.$ and $\left.\left(\sqrt{\overline{v^{\prime 2}}}\right)_{d, \text { int }} / U_{d, \text { int }} \approx 0.18\right]$, than for grassed floodplains $\left[\left(\sqrt{\overline{u^{\prime 2}}}\right)_{d, \text { int }} / U_{d, \text { int }} \approx 0.23\right.$ and $\left.\left(\sqrt{\overline{v^{\prime 2}}}\right)_{d \text {,int }} / U_{d, \text { int }} \approx 0.11\right]$. These values are independent of water depth and lateral mass transfers. Figure 16 therefore suggests that there is an equilibrium within the inner organisation of the structure between convection velocity of the structure and amplitude of the velocity fluctuations within 

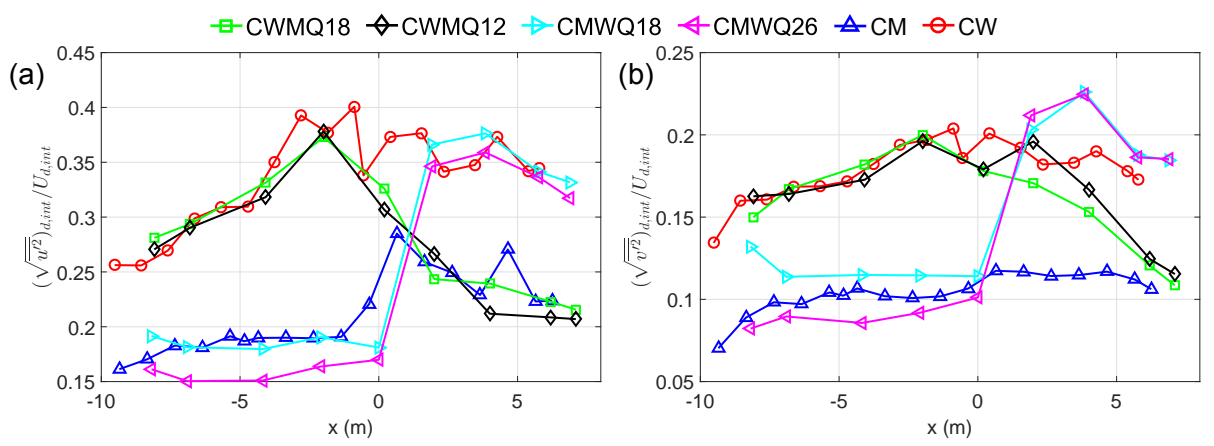

Fig. 16: Longitudinal evolution of (a) the longitudinal and (b) the lateral depthaveraged turbulence intensities at the interface, normalised by the depth-averaged velocity.

the structure. In the region downstream of the transition, an adjustment length is observed before the equilibrium related to the new roughness is reached. This adjustment length is higher for the wood-to-meadow $(\approx 5 \mathrm{~m})$ than for the meadow-to-wood transition $(\approx 3 \mathrm{~m})$.

We propose the following process for explaining the mixing layer dynamics. When the ratio $\left(\sqrt{\overline{u^{\prime 2}}}\right)_{d, \text { int }} / U_{d, \text { int }}\left(\right.$ or $\left.\left(\sqrt{\overline{v^{\prime 2}}}\right)_{d, \text { int }} / U_{d, \text { int }}\right)$ is out of equilibrium, the value of $\left(\sqrt{\overline{u^{\prime 2}}}\right)_{d, i n t}$ varies in order to reach equilibrium (the value of $U_{d, \text { int }}$ being determined macroscopically). This in turn induces a change in the coherent structure size according to the conservation law of angular momentum (Section 6.2), and therefore a change in mixing layer width.

\section{Turbulent exchange at the interface}

The modelling of the turbulent exchange at the main channel/floodplain interface is a key issue in the numerical modelling of compound channel flows. Figure 17a shows the depth-averaged lateral Reynolds stresses at the interface normalised by the depth-averaged velocity $U_{d, i n t}$. The ratio $\overline{u^{\prime} v^{\prime}} d$,int $/ U_{d, i n t}^{2}$ appears to be rather constant and little dependent on flow conditions for a given roughness type, as it is the case for the turbulence intensities (Section 6.3). This ratio is much higher for the wooded floodplain $\left(\overline{u^{\prime} v^{\prime}} d\right.$,int $\left./ U_{d, i n t}^{2}=0.040-0.050\right)$ than for the grassed floodplain $\left(\overline{u^{\prime} v^{\prime}}{ }_{\text {,int }} / U_{d, \text { int }}^{2}=0.005-0.015\right)$, indicating that turbulence production and therefore turbulence exchange are enhanced in the presence of emergent cylinders on the floodplains. An adjustment length downstream of the transition is required for reaching the level related to the downstream roughness. Similarly to the normalised turbulence intensities (Fig. 16), this adjustment length is higher for the wood-to-meadow $(\approx 6 \mathrm{~m})$ than for the meadow-to-wood transition $(\approx 4 \mathrm{~m})$. An adjustment length is also observed immediately downstream of the channel inlet, especially when the floodplains are covered with wood (CW and CWM).

In several compound channel 1D numerical models (Bousmar and Zech, 1999; Proust et al, 2009) the lateral shear stresses at the interface are modelled with 

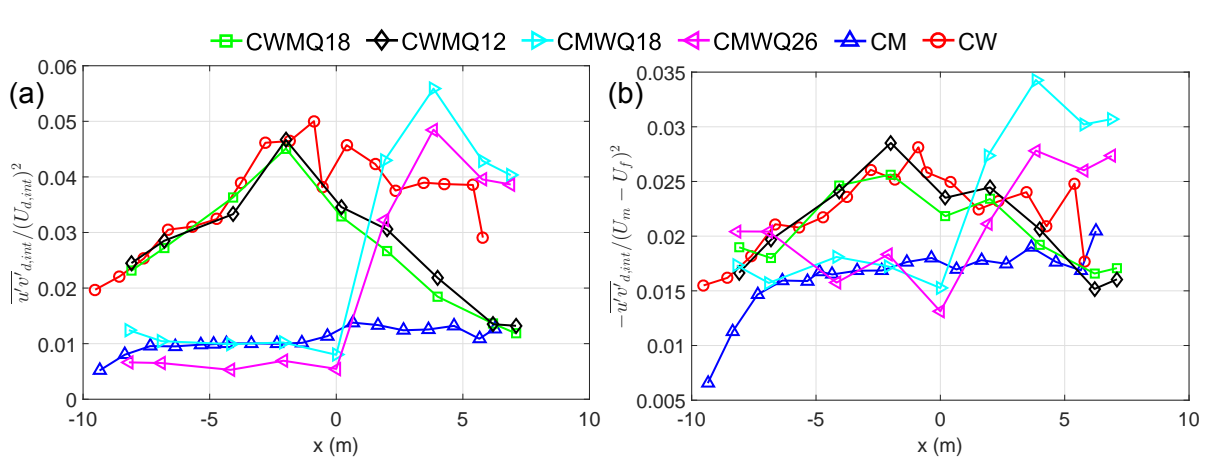

Fig. 17: Depth-averaged lateral Reynolds stresses at the interface normalised (a) by $U_{d, \text { int }}$ and (b) by the bulk velocity difference between subsections $U_{m}-U_{f}$.

the formula $\overline{u^{\prime} v^{\prime}}{ }_{d, i n t}=\Psi_{t}\left(U_{m}-U_{f}\right)^{2}$, where $\Psi_{t}$ is a calibrating parameter that is assumed to be constant. Figure $17 \mathrm{~b}$ shows the ratio $\overline{u^{\prime} v^{\prime}} d$,int $/\left(U_{m}-U_{f}\right)^{2}$ for the various test cases. If the upstream flow development of test case CM between $x=-9$ and $x=-7 \mathrm{~m}$ is not taken into account (probably affected by the inlet conditions), the ratio varies between 0.013 and 0.034 for all flow cases. The mean value is close to that used by Proust et al (2009), i.e. $\Psi_{t}=0.020$. However, similarly to the ratio $\overline{u^{\prime} v^{\prime}} d$,int $/ U_{d, \text { int }}^{2}$, the coefficient $\Psi_{t}$ seems to be specific for a roughness type: $\Psi_{t} \approx 0.017$ for grassed floodplains and $\Psi_{t} \approx 0.025$ for wooded floodplains.

Figure 17a suggests that $U_{d, \text { int }}$ is more appropriate to normalise the lateral shear stress. However, the macroscopic quantity $U_{m}-U_{f}$ is easier to compute in numerical models and seems to give acceptable results.

\section{Secondary currents}

In the case of non-uniform flows in straight compound channels, both lateral shear stress and secondary currents can contribute to the lateral exchange of longitudinal momentum (Proust et al, 2013). The depth-averaged lateral exchange of longitudinal momentum due to secondary currents is given by $\rho\left(U\left(V-V_{d}\right)\right)_{d}$, where subscript $d$ stands for depth-averaging (Vermaas et al, 2011). Figure 18 shows the lateral distribution of this quantity in the main channel. Profiles upstream and downstream of the transition are shown for test cases CWMQ18 and CMWQ18, along with the uniform flow test cases CM and CW. For the test cases with a roughness transition, the momentum transfers due to secondary currents are more important in reaches with wooded floodplains (dashed lines) than in reaches with grassed floodplains. The lateral exchange of momentum by secondary currents is equal to zero at the main channel centreline, which is expected owing to flow symmetry. The quantity $\rho\left(U\left(V-V_{d}\right)\right)_{d}$ is also weak at the interface with the floodplain, at least compared with the lateral shear stress that is on the order of $3 \mathrm{~Pa}$ for example for test case $\mathrm{CM}$ (Fig. 17, with $U_{d, \text { int }} \approx 52 \mathrm{~cm} \cdot \mathrm{s}^{-1}$ for $\mathrm{CM}$ ). Secondary currents thus generate a redistribution of momentum inside the main channel (momentum is transferred positively in the direction of the floodplain), but the exchange with the floodplains appears to be weak. 


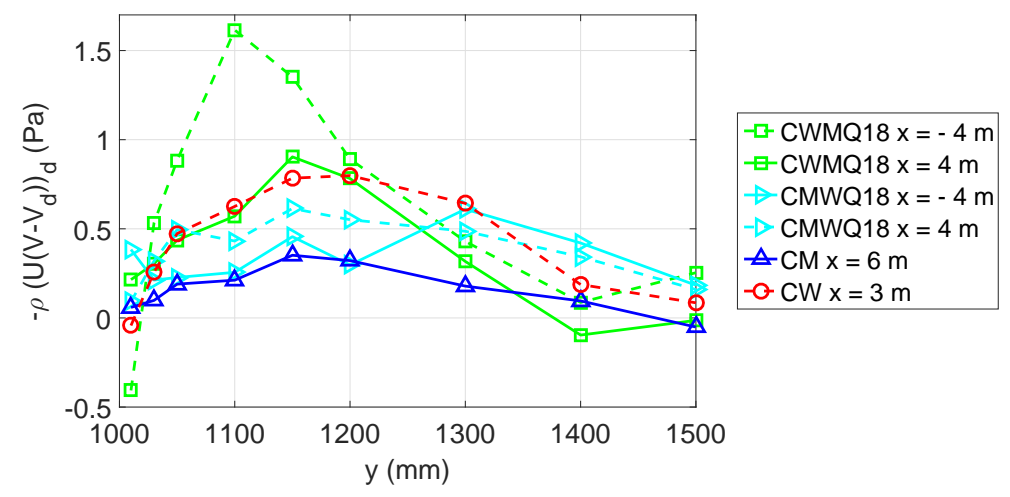

Fig. 18: Lateral distribution of the depth-averaged lateral transfer of longitudinal momentum due to secondary currents in the main channel. Dashed lines denote profiles within reaches with wooded floodplains.

Figure 19a shows the longitudinal evolution $\rho\left(U\left(V-V_{d}\right)\right)_{d}(x)$ along the position $y=1150 \mathrm{~mm}$, i.e. where this quantity is nearly maximum in the width (see Fig. 18). Over a uniform roughness (test cases CM and CW), the quantity $\rho\left(U\left(V-V_{d}\right)\right)_{d}$ is rather constant after $x=-1 \mathrm{~m}$ and is higher for the wooded floodplain than for the grassed floodplain. Accordingly, a decrease of the momentum exchange due to secondary currents $\rho\left(U\left(V-V_{d}\right)\right)_{d}$ is observed downstream of the wood-to-meadow transition and an increase is observed downstream of the meadow-to-wood transition.

Figure 19b shows the same quantity as in Fig. 19a, but normalised by $U_{d, \text { int }}$. After an adjustment length, both downstream of the channel inlet and downstream of the roughness transition, the quantity $\left(U\left(V-V_{d}\right)\right)_{d} / U_{d, i n t}^{2}$ is quasi invariant for a given floodplain roughness, irrespective of flow conditions. The value is nearly four times higher for the wooded floodplain than for the floodplain covered by a meadow, such that the presence of emergent cylinders on the floodplain enhances the secondary currents in the main channel.

The equilibrium between the magnitude of the secondary currents and the convection velocity of the horizontal structures $U_{d, i n t}$ suggests an interaction between secondary currents and horizontal structures in compound channel flows, an assumption that was already put forward by previous authors (Nezu and Nakayama, 1997; Lukowicz, 2002). Nezu and Nakayama (1997) suggested that secondary currents are indeed intermittent vortical structures that are coupled with the primary horizontal vortices, forming a single complex 3D structure. Further studies are required to clarify this issue, especially the geometrical arrangement of this macro-structure and the dynamical interaction between secondary currents and horizontal vortices.

\section{Conclusion}

The study experimentally investigated compound channel flows with a longitudinal roughness transition on the floodplains between a bed roughness, modelled by a plastic grass (grassed floodplains), and emergent macro-roughnesses, modelled by an array of emergent cylinders installed on a rough bed (wooded floodplains), and 

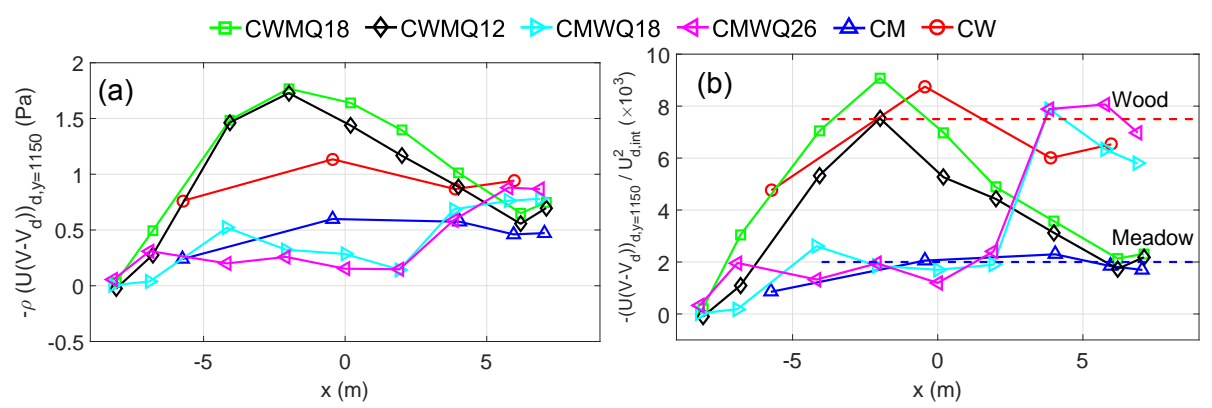

Fig. 19: Longitudinal variation in the depth-averaged lateral transfer of longitudinal momentum due to secondary currents at $y=1150 \mathrm{~mm}$; (a) dimensional values and (b) values normalised by $U_{d, i n t}$.

vice versa. These flows were compared to uniform compound channel flows with only grassed or only wooded floodplains.

Contrary to longitudinal roughness transitions in a single rectangular channel, for which changes in flow depth are observed only upstream of the roughness transition (Dupuis et al, 2016), in a compound channel configuration the water depth can vary all along the flume. This can be related to the lateral mass exchange between subsections that represents a supplementary degree of freedom, compared to the single channel.

Irrespective of flow conditions, the lateral profiles of mean longitudinal velocity feature an inflection point that is located very near the main channel/floodplain interface. The centre of the mixing layer that forms at the interface between subsections, is defined at the inflection point.

The mean velocity at the interface (at the inflection point) $U_{\text {int }}$ appeared to play a key role in the flow processes:

1. As the mixing layer evolves differently from either side of the interface, the mixing layer width was divided into two parts: one in the main channel, the other in the floodplain. At a given altitude, when these two widths are normalised by the floodplain water depth and by a velocity difference scaled by $U_{\text {int }}$, two plateau values are observed, each value being related to a floodplain roughness type. The plateau value is about three times higher for reaches with grassed floodplains than with wooded floodplains, indicating that the cylinder array limits the transverse development of the mixing layer.

2. At a given altitude (mid-depth of the floodplain flow), the mean velocity profiles across the mixing layer, normalised with $U_{\text {int }}$ and with the subsection mixing layer widths, are superimposed, irrespective of downstream position, flow depth, floodplain roughness type, and lateral mass transfers. By contrast, turbulent quantities profiles do not coincide, showing that the flows are not fully self-similar and that the eddy viscosity assumption cannot be used in this case.

3. For a given floodplain roughness type, the turbulence intensities and the lateral Reynolds stresses at the interface scale with the depth-averaged velocity at the interface $U_{d, i n t}$. Moreover, these turbulence quantities normalised by $U_{d, i n t}$ are about three times higher for the wooded floodplain than for the grassed flood- 
plain, indicating that turbulence production is higher in the presence of emergent cylinders on the floodplains.

4. For a given floodplain roughness type, the magnitude of the secondary currents in the main channel also scales with $U_{d, i n t}$. The lateral flux of momentum due to secondary currents normalised by $U_{d, i n t}$ is about four times higher with wooded floodplains than with grassed floodplains.

The fact that both the turbulent quantities and the secondary current magnitude scale with $U_{d, i n t}$ (which was shown to be the convection velocity of the structures by Dupuis et al, 2017), tends to support the hypothesis that primary vortices with vertical axis and secondary currents with longitudinal axis are coupled and form a unique 3D vortical structure (Nezu and Nakayama, 1997). We suggest that this vortical structure tend to a state where intensity of the velocity fluctuations (of both horizontal vortices and secondary currents) and spatial extension of the structure are in equilibrium. Downstream of the change in roughness, an adjustment length is observed before the coherent structure equilibrium relative to the new roughness is achieved, i.e. before normalised velocity fluctuations, secondary currents intensity and mixing layer width reach the value corresponding to the new roughness.

The lateral mass transfers have no influence on the normalised values of turbulence intensities, Reynolds stresses, mixing layer widths, and secondary currents intensity. This suggests that the proposed normalisation by $U_{\text {int }}$ accurately takes into account the effect of the lateral net mass exchanges on the flow structure.

The analysis of the autocorrelation function shows that the length of the coherent structures is proportional to the total width of the mixing layer, irrespective of flow conditions and of downstream position.

Two different processes are identified in the evolution dynamics of the coherent structures. First, vortex merging and/or fluid entrainment are responsible for the overall growth of the structures all along the flume. Second, a mechanism that conserves angular momentum (e.g. vortex stretching) is additionally involved downstream of the roughness transition in order to adapt (increase or decrease) the structure size to the new flow conditions.

Acknowledgements The $\mathrm{PhD}$ grant of $\mathrm{V}$. Dupuis was funded by Irstea and by the French National Research Agency (Flowres project, grant No. ANR-14-CE03-0010, https://flowres.irstea.fr/en/). The authors greatly thank Fabien Thollet and Alexis Buffet for their technical support.

\section{Appendix}

The momentum equation of the non-uniform straight compound channel flow, subsection-averaged in the main channel, reads (see e.g. Proust et al, 2009):

$$
\begin{array}{r}
S_{0}=\frac{1}{g H_{m}} \frac{\partial H_{m} U_{m}^{2}}{\partial x}-\frac{U_{d, i n t}}{g H_{m}} \frac{\partial H_{m} U_{m}}{\partial x}+\frac{\partial H_{m}}{\partial x} \\
-\frac{2 H_{f}\left(\overline{u^{\prime} v^{\prime}}\right)_{y=y_{i n t}, z>z_{B F}, d}}{g H_{m} B_{m}}-\frac{\left(\overline{u^{\prime} w^{\prime}}\right)_{z=0, w}}{g H_{m}}-\frac{2 z_{B F}\left(\overline{u^{\prime} v^{\prime}}\right)_{y=y_{i n t}, z<z_{B F}, d}}{g H_{m} B_{m}}
\end{array}
$$

where $S_{0}$ is the bottom slope, $H_{m}$ the main channel flow depth, $H_{f}$ the floodplain flow depth, $B_{m}$ the main channel width, $z_{B F}$ the main channel bankfull level, $U_{m}$ the main 
channel bulk velocity, subscript $d$ stands for depth-averaging, subscript $w$ stands for width-averaging and $y_{\text {int }}$ is the lateral coordinate of the main channel/floodplain interface.

In Eq. 11, the gravitational force (bottom slope) is balanced by (in the order of the terms on the right-hand side of the equation): (1) the longitudinal flux of momentum, (2) the lateral net mass exchange with the floodplains, (3) the longitudinal pressure gradient, (4) the turbulent exchange at the interface, (5) the bed friction, (6) the friction on the lateral walls (beneath the bankfull level).

Similarly, the momentum equation subsection-averaged in the right-hand floodplain reads:

$$
\begin{array}{r}
S_{0}=\frac{1}{g H_{f}} \frac{\partial H_{f} U_{f}^{2}}{\partial x}-\frac{U_{d, i n t}}{g H_{f}} \frac{\partial H_{f} U_{f}}{\partial x}+\frac{\partial H_{f}}{\partial x} \\
+\frac{\left(\overline{u^{\prime} v^{\prime}}\right)_{d, y=y_{i n t}, z>z_{B F}}}{g B_{f}}-\frac{\left(\overline{u^{\prime} w^{\prime}}\right)_{z=z_{B F}, w}}{g H_{f}}+\frac{a C_{D} U_{f}^{2}}{2 g} .
\end{array}
$$

where $U_{f}$ is the floodplain bulk velocity, $B_{f}$ the floodplain width. The last term on the right-hand side stands for the drag forces of the cylinder array, where $a$ is the frontal area per unit volume $(a=N D)$ and $C_{D}$ is the cylinder drag coefficient.

The momentum flux term in Eq. 11 or 12 can be split into two terms:

$$
\frac{1}{g H_{i}} \frac{\partial H_{i} U_{i}^{2}}{\partial x}=\frac{2 U_{i}}{g H_{i}} \frac{\partial H_{i} U_{i}}{\partial x}-\frac{U_{i}^{2}}{g H_{i}} \frac{\partial H_{i}}{\partial x}
$$

with $i \in\{m, f\}$. The first term is the acceleration/deceleration due to positive/negative lateral mass exchange and the second term is the acceleration/deceleration due to the contraction/expansion of the flow through water depth variation. The factor 2 before the first term can be interpreted as follows. Consider a positive lateral mass transfer without water depth variation: on the one hand the flow has to be "compressed" by the entering lateral flow, i.e. accelerated in order to let place for the entering flow; on the other hand, the arriving flow has to be accelerated/decelerated to reach the subsection velocity. Flow leaving the subsection produces similar effects.

When the first term on the right-hand side of Eq. 13 is combined with the mass exchange term of Eq. 11 or 12 (first term on the right-hand side), the normalised total force exerted by the lateral mass exchange is obtained:

$$
A_{i}=\frac{2 U_{i}-U_{d, i n t}}{g H_{i}} \frac{\partial H_{i} U_{i}}{\partial x}
$$

\section{References}

Bousmar D, Zech Y (1999) Momentum transfer for practical flow computation in compound channels. Journal of hydraulic engineering 125(7):696-706

Bousmar D, Wilkin N, Jacquemart JH, Zech Y (2004) Overbank flow in symmetrically narrowing floodplains. Journal of hydraulic engineering 130(4):305-312

Bousmar D, Riviere N, Proust S, Paquier A, Morel R, Zech Y (2005) Upstream discharge distribution in compound-channel flumes. Journal of Hydraulic Engineering 131(5):408-412 
Brown GL, Roshko A (1974) On density effects and large structure in turbulent mixing layers. Journal of Fluid Mechanics 64(04):775-816

Chu VH, Babarutsi S (1988) Confinement and bed-friction effects in shallow turbulent mixing layers. Journal of hydraulic engineering 114(10):1257-1274

Dupuis V (2016) Experimental investigation of flows subjected to a longitudinal transition in hydraulic roughness in single and compound channels. PhD thesis, Université de Lyon

Dupuis V, Proust S, Berni C, Paquier A (2016) Combined effects of bed friction and emergent cylinder drag in open channel flow. Environmental Fluid Mechanics 16(6):1173-1193

Dupuis V, Proust S, Berni C, Paquier A (2017) Mixing layer development in compound channel flows with submerged and emergent rigid vegetation over the floodplains. Experiments in Fluids

Elliott S, Sellin R (1990) SERC flood channel facility: skewed flow experiments. Journal of Hydraulic Research 28(2):197-214

Fjortoft R (1950) Application of integral theorems in deriving criteria of stability for laminar flows and for the baroclinic circular vortex. Geofys Publ 17(6):1-52

George WK (1989) The self-preservation of turbulent flows and its relation to initial conditions and coherent structures. Advances in turbulence pp 39-73

Goring DG, Nikora VI (2002) Despiking acoustic Doppler velocimeter data. Journal of Hydraulic Engineering 128(1):117-126

Jahra F, Kawahara Y, Hasegawa F, Yamamoto H (2011) Flow-vegetation interaction in a compound open channel with emergent vegetation. International journal of river basin management 9(3-4):247-256

Knight DW, Demetriou JD (1983) Flood plain and main channel flow interaction. Journal of Hydraulic Engineering 109(8):1073-1092

Lukowicz Jv (2002) Zu kohärenten Turbulenzstrukturen in der Strömung gegliederter Gerinne. PhD thesis, RWTH Aachen

Moore D, Saffman P (1975) The density of organized vortices in a turbulent mixing layer. Journal of Fluid Mechanics 69(03):465-473

Nezu I, Nakayama T (1997) Space-time correlation structures of horizontal coherent vortices in compound open-channel flows by using particle-tracking velocimetry. Journal of Hydraulic Research 35(2):191-208

Nikora V, McEwan I, McLean S, Coleman S, Pokrajac D, Walters R (2007) Doubleaveraging concept for rough-bed open-channel and overland flows: Theoretical background. Journal of Hydraulic Engineering 133(8):873-883

Peltier Y, Proust S, Riviere N, Paquier A, Shiono K (2013) Turbulent flows in straight compound open-channel with a transverse embankment on the floodplain. Journal of Hydraulic Research 51(4):446-458

Proust S, Bousmar D, Riviere N, Paquier A, Zech Y (2009) Nonuniform flow in compound channel: A 1-d method for assessing water level and discharge distribution. Water resources research $45(12)$

Proust S, Bousmar D, Rivière N, Paquier A, Zech Y (2010) Energy losses in compound open channels. Advances in water Resources 33(1):1-16

Proust S, Fernandes JN, Peltier Y, Leal JB, Riviere N, Cardoso AH (2013) Turbulent non-uniform flows in straight compound open-channels. Journal of Hydraulic Research 51(6):656-667

Rominger JT, Nepf HM (2011) Flow adjustment and interior flow associated with a rectangular porous obstruction. Journal of Fluid Mechanics 680:636-659 
Tominaga A, Nezu I (1991) Turbulent structure in compound open-channel flows. Journal of Hydraulic Engineering 117(1):21-41

Townsend A (1961) Equilibrium layers and wall turbulence. Journal of Fluid Mechanics 11(01):97-120

Vermaas D, Uijttewaal W, Hoitink A (2011) Lateral transfer of streamwise momentum caused by a roughness transition across a shallow channel. Water resources research $47(2)$

White BL, Nepf HM (2007) Shear instability and coherent structures in shallow flow adjacent to a porous layer. Journal of Fluid Mechanics 593:1-32

Winant C, Browand F (1974) Vortex pairing: the mechanism of turbulent mixing-layer growth at moderate reynolds number. Journal of Fluid Mechanics 63(02):237-255

Zong L, Nepf H (2010) Flow and deposition in and around a finite patch of vegetation. Geomorphology 116(3):363-372 